\title{
Evaluation and Optimisation of Interface Force Fields for Water on Gold Surfaces
}

\author{
Andrej Berg, ${ }^{\dagger}$ Christine Peter, ${ }^{\dagger}$ and Karen Johnston*, ${ }^{*}$ \\ $\dagger$ Department of Chemistry, University of Konstanz, Universitätsstraße 10, Konstanz \\ 78457, Germany \\ $\ddagger$ Department of Chemical and Process Engineering, University of Strathclyde, James Weir \\ Building, 75 Montrose Street, Glasgow G1 1XJ, United Kingdom \\ E-mail: karen.johnston@strath.ac.uk \\ Phone: +44 (0)141548 4084
}

\begin{abstract}
The structure and dynamics of water at gold surfaces is important for a variety of applications, including lab on a chip and electrowetting. Classical molecular dynamics (MD) simulations are frequently used to investigate systems with water-gold interfaces, such as biomacromolecules in gold nanoparticle dispersions, but the accuracy of the simulations depends on the suitability of the force field. Density functional theory (DFT) calculations of a water molecule on gold were used as a benchmark to assess force field accuracy. It was found that Lennard-Jones potentials did not reproduce the DFT water-gold configurational energy landscape whereas the softer Morse and Buckingham potentials allowed for a more accurate representation. MD simulations with different force fields exhibited rather different structural and dynamic properties of water on a gold surface. This emphasises the need for experimental data and further effort on the validation of a realistic force field for water-gold interactions.
\end{abstract}




\section{Introduction}

Solid-liquid interfaces play an important role in a huge variety of technologies, such as functionalised nanoparticles for drug delivery, heterogeneous nucleation and crystal growth in pharmaceutical development and manufacture, and surface wetting in self-cleaning or anti-fouling surfaces. Water is a key component of all these systems, and rational design of these technologies necessitates a thorough understanding of the interaction of water with surface and functional groups. On the other hand, the importance of gold as a functionalised material has increased in recent decades, due to the use of gold nanoparticles in biological systems ${ }^{1}$ or the use of gold surfaces for plasmon resonance. Studies of wetting of water droplets on gold surfaces is important in applications such as nanofluidic manipulation, lab on a chip and electrowetting. ${ }^{2,3}$

Classical molecular dynamics (MD) simulations are frequently employed due to their ability to provide molecular-level insight into these applications and explore underlying mechanistic details. Furthermore, this computational method allows the examination of a broad time range and system size by the use of different levels of resolution. ${ }^{4}$ However, the ability of MD simulations to accurately predict the behaviour of such systems, depends on the suitability of the force field. Force fields are sets of parameters for various potential energy functions $(\mathrm{PEF})$ which describe the interactions inside the simulated system.

Force fields for homogeneous systems, such as liquids or bulk crystals, are often parameterised by fitting simulation results to available experimental thermodynamic data ${ }^{5,6}$ or to quantum mechanics calculations. ${ }^{7}$ Changing the force field can yield significantly different results for the same system. For example, a study of protein folding using four different force fields found that while the rate of folding was similar in each case the folding mechanism and

properties of the unfolded state depended substantially on the force field. ${ }^{8}$ Another example is a study of amino acid solutions that compared eight different force field combinations and, while there were similarities in some properties, such as density, other properties were qualitatively different. ${ }^{9}$ 
Force fields are often parameterised to describe an individual molecule type in a particular environment. For mixed systems with several different molecule types in this environment, it is common to take force field parameters developed for the individual molecules and use mixing rules to obtain the desired force field parameters for the mixed molecular system. This approach is well known for biological systems, like proteins, where force field parameters are obtained from a set of homotypic interaction potentials. However, force field parameters that were derived for molecules in one environment, e.g. aqueous solution, will not necessarily represent the behaviour of the same molecules in different environments, such as liquid-solid heterogeneous systems.

To address heterogeneous systems, there have been several recent developments of interfacespecific force fields. One example is the INTERFACE force field that was developed specifically to describe the interaction of soft matter with a variety of inorganic surfaces. ${ }^{10}$ It is based on Lennard-Jones (LJ) potentials for easy compatibility with standard organic and biomolecular force fields via a mixing rule approach to obtain solid-soft matter interactions. The LJ parameters and partial charges were chosen to describe measured bulk and interfacial properties, such as density and surface tension.

However, reliable and detailed experimental reference data is not always available, and an alternative approach is required. One approach is to use ab initio molecular dynamics (AIMD) simulations but this is limited to small system sizes and short simulation times. ${ }^{11-13}$ Another approach is to develop force fields for solid-liquid interfaces, using quantum mechanics calculations to obtain benchmark data and then using an optimisation algorithm to parametrise the force field. This has been done for a range of materials including organic molecules on titania, ${ }^{14}$ amino acids on gold ${ }^{15,16}$ (GolP force field), water on zinc oxide, ${ }^{17}$ benzene on gold ${ }^{18}$ and ethanol on alumina, ${ }^{19}$ just to name some examples.

While other studies have focused on optimising the force field parameters for the interaction of amino acids ${ }^{16}$ or nucleobases ${ }^{20}$ on gold, in this work, we focus on the investigation of the water-gold interface. Our aims in this work are threefold: 1) to compare the available 
force fields for water-gold interfaces, 2) to develop an improved force field and 3) to test the impact of the force field on structural and dynamic properties of water next to a gold interface. Existing force field parameters from literature were assessed based on their ability to reproduce interaction energies from density functional theory (DFT) calculations. Force field optimisation is done by matching classical interaction energies to DFT results for several types of PEFs. Furthermore, we test whether the force field is transferable from the $\mathrm{Au}(111)$ surface to the $\mathrm{Au}(001)$ surface, and vice versa, which is an an important property for the study of gold nanoparticles in aqueous environments Finally, our results are evaluated by comparison of classical MD simulations with AIMD results. ${ }^{21}$

\section{Computational Details}

Density functional theory calculations were performed with PWSCF within the Quantum ESPRESSO package ${ }^{22}$ version 5.1. All DFT calculations which were used for comparison with classical models were performed using the vdW-DF ${ }^{23-25}$ exchange and correlation functional with a plane-wave cutoff of 50 Ry and a charge density cutoff of 400 Ry. MarzariVanderbilt cold smearing ${ }^{26}$ was applied for Brillouin-zone integration using a smearing width of $0.02 \mathrm{Ry}$. To investigate the influence of vdW-DF on the interaction of water at the gold surface selected calculations were repeated with the $\mathrm{PBE}^{27}$ exchange and correlation functional. In all cases core electrons were described using ultrasoft pseudopotentials available from the Quantum ESPRESSO website (see SI). For all ionic relaxations the force convergence criterion was $0.051 \mathrm{eV} / \AA$.

Two surfaces of gold were constructed from corresponding unit cells which were obtained by ionic relaxations of bulk gold using the vdW-DF functionals. The cubic unit cell for the $\mathrm{Au}(001)$ surface contained four atoms with a lattice constant of $a=0.426 \mathrm{~nm}$ while the hexagonal unit cell for the $\mathrm{Au}(111)$ surface contained three atoms with lattice constants of $a=0.301 \mathrm{~nm}$ and $c=0.737 \mathrm{~nm}$. Surfaces for calculation of interaction energies were 
constructed from four layers of atoms with periodic boundary conditions and a distance of $1.9 \mathrm{~nm}$ for the vacuum which was found to be large enough for this setup by increasing the cell in the $z$-direction until no energy difference was observed. The $\mathrm{Au}(001)$ surface was a $3 \times 3$ super cell with 72 gold atoms and a k-point grid of $2 \times 2 \times 3$ while the $\mathrm{Au}(111)$ surface was a $4 \times 4$ super cell with 64 atoms and a k-point grid of $2 \times 2 \times 2$.

The two upper atomic layers of bare gold surfaces were relaxed by vdW-DF while the rest of the gold atoms were held fixed. A single water molecule was placed on top of these optimised surfaces and interaction energies, $E_{\text {int }}$, were calculated by a single self-consistent field calculation without any further ionic relaxation. Additionally, the lowest energy metastable configurations of water on each gold surface and top, hollow and bridge adsorption sites were obtained by relaxing the water molecule but keeping the surface fixed. These configurations have adsorption energies, $E_{\text {ads }}$.

Classical force field energy calculations and the force field optimisation were performed using the GULP software. ${ }^{28}$ The same structures were used in both the quantum and classical calculations of interaction and adsorption energies. Classical calculations used a cut-off of $1.2 \mathrm{~nm}$. Parameter optimisation of classical pair potentials was done using the BroydenFletcher-Goldfarb-Shanno (BFGS) algorithm, ${ }^{29,30}$ implemented in GULP.

All MD simulations and subsequent analysis were performed using the GROMACS package, version 5.0.7. ${ }^{31}$ For all non-bonded interactions a cut-off of $1.2 \mathrm{~nm}$ was used. For LJ 12-6 potentials the cut-off was treated by a switch function between $1.0-1.2 \mathrm{~nm}$ while for all other potential types which were implemented in a tabulated form this was done by a shift function. The PME electrostatic scheme ${ }^{32}$ was applied with a cut-off of $1.2 \mathrm{~nm}$. The time step was $2 \mathrm{fs}$ at a constant temperature of $300 \mathrm{~K}$ using a velocity rescale thermostat. ${ }^{33}$ We modelled the $\mathrm{Au}(001)$ and $\mathrm{Au}(111)$ surface with a size of $4.88 \mathrm{~nm} \times 4.88 \mathrm{~nm}$ and $5.20 \mathrm{~nm} \times 5.20 \mathrm{~nm}$ in the $x$ and $y$-directions, respectively, and a thickness of eight atoms separated by $4.5 \mathrm{~nm}$ of water. For all simulations gold was modelled as neutral LJ particles with interaction parameters published for LJ 12-6 potentials by Heinz et al. without any 
further constrains. ${ }^{34}$ Simulations with force fields from literature were performed using the $\mathrm{SPC} / \mathrm{E},{ }^{35} \mathrm{mTIP}^{3} \mathrm{P}^{36}$ and $\mathrm{COMPASS}^{7}$ water models. Our own force fields were used in combination with the SPC/E water model only. Initial conformations for MD simulations were obtained by energy minimisation and two subsequent 200 ps equilibration runs under NVT and NPT conditions. For NPT equilibration pressure was held constant at 1 bar using the Parrinello-Rahman approach. ${ }^{37}$

\section{Results}

In this section we first present DFT interaction energies for a representative set of configurations for a water molecule on the $\mathrm{Au}(001)$ and $\mathrm{Au}(111)$ surfaces and test the influence of the vdW-DF functional on interaction energies of different orientations of water on the surface. This set of configurations is used to benchmark the performance of four force fields from the literature by comparing how well they reproduce the DFT interaction energies. We then use the following approaches to find the types of pair potentials and best parameterisation that minimise the difference between force field and DFT interaction energies: 1) optimise only the Au LJ potential from which all gold-water interactions are derived via standard mixing rules, 2) optimise the gold-water LJ interaction potentials independently (without the use of mixing rules), and 3) change the functional form of the potential and optimise the parameters. Finally, we present structural and dynamical properties of a thin film of water confined between two gold surfaces for several force fields.

\subsection{Density functional theory calculations}

First we present adsorption energies for metastable configurations $E_{\text {ads }}$ of water on both the (111) and (001) gold surfaces, and on the top, bridge and hollow sites. On each adsorption site the water molecule was allowed to ionically relax to a metastable configuration with the 
gold atoms held fixed. The interaction energy $E_{\text {int }}$ of a water molecule on gold is defined as:

$$
E_{\text {int }}=E_{\text {total }}-\left(E_{\text {surf }}+E_{\text {mol }}\right)
$$

where $E_{\text {total }}$ is the total energy of the system, $E_{\text {surf }}$ is the energy of the bare surface, and $E_{\text {mol }}$ is the energy of a single water molecule. Following the work of Nadler and Sanz we can describe the orientation of water molecules relative to the the gold surface via two angles, $\Phi$ and $\Psi$. Angle $\Phi$ is between the water molecule dipole and the surface normal, and $\Psi$ is between the normal of the water molecule plane and the surface normal (Fig. 1). ${ }^{21}$ This means that a water molecule which is adsorbed exactly flat on the surface has values of $\Phi=$ $90^{\circ}$ and $\Psi=0^{\circ}$.

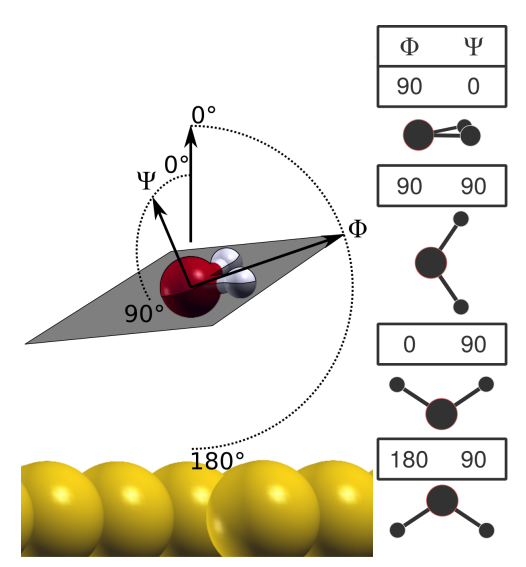

Figure 1: Angles to describe orientation of a water molecule in respect of a surface. $\Phi$ is the angle between the water molecule dipole and the surface normal. $\Psi$ describes the angle between the normal of the water molecule plane and the surface normal. Right panel shows examples for possible orientations on top of a surface and the corresponding values for $\Phi$ and $\Psi$.

The adsorption energies and orientations of water on the various surface sites are presented in Table 1. For both (111) and (001) surfaces, the lowest energy conformation was a nearly flat water molecule with the oxygen atom on top of a gold atom. This preferred adsorption mode of water oxygen on top of noble metal surfaces was observed in various studies and reviewed by Verdaguer et al. ${ }^{38}$ In a recent combined X-ray adsorption spectroscopy and AIMD study by Velasco-Velez et al. it was also shown that for liquid water on gold the flat 
orientation of interfacial water is preferred. ${ }^{39}$ In all cases vdW-DF calculations yielded lower energies than PBE functionals without vdW interactions. Adsorption energies for the top site on $\mathrm{Au}(111)$ were obtained by Nadler and Sanz in an extended study on the influence of DFT functionals on the adsorption energy. Our PBE result of $-16.5 \mathrm{eV}$ is lower than their result of $-10.6 \mathrm{eV}$, which could be attributed to methodological differences. vdW interactions significantly lowered this adsorption energy to -23.3 using vdW-DF, which is in reasonable agreement with Nadler and Sanz' optB86b-vdW value of -28.9. Our PBE results for the $\mathrm{Au}(111)$ top site are also in reasonable agreement with those of other GGA functionals. ${ }^{40,41}$ We can also compare our vdW-DF results on the $\mathrm{Au}(001)$ surface with the results of Wright et al.. ${ }^{16}$ Although our results for the $\mathrm{Au}(001)$ surface have lower $E_{\text {ads }}$ compared to Wright et al. adsorption sites can be ordered energetically in the same way with the top site being lowest energy followed by bridge then hollow. The adsorption energies are later used for validation regarding the ability of a classical model to reproduce the correct lowest energy adsorption site on a gold surface.

We aim to use a set of DFT interaction energies for assessing the accuracy of classical force fields and for fitting. Therefore, DFT calculations were performed for 174 configurations of a water molecule in various orientations and at different distances on top, bridge and hollow sites on the $\mathrm{Au}(111)$ and $\mathrm{Au}(001)$ surfaces. 88 calculations were performed on the $\mathrm{Au}(001)$ and 86 calculations on the $\mathrm{Au}(111)$ surface. These configurations were chosen to give a representative sample of water on a surface, so that some molecules were oriented with the oxygen atom closer to the Au surface and others with one or both hydrogen atoms closer to the surface and a distribution of low and high energy configurations. All interaction energies were obtained by performing self consistent field (scf) calculations without any ionic relaxation.

The distance-dependent interaction energies of two distinct orientations of water on the $\mathrm{Au}(001)$ top site are shown in Figure 2. For these configurations, the scf calculations were repeated using the PBE functional to show the influence of vdW forces. Configurations where 
Table 1: DFT adsorption energies of water on the $\mathrm{Au}(001)$ and $\mathrm{Au}(111)$ surface for different functionals and the lowest energy configurations found by other studies. Adsorption energies are in $\mathrm{kJ} \mathrm{mol}^{-1}$, distance in $\mathrm{nm}$ and angles in degrees.

\begin{tabular}{|c|c|c|c|c|c|c|c|}
\hline Functional & Surface & Site & $E_{a d s}$ & $d_{\mathrm{Au}-\mathrm{O}}$ & $\Phi$ & $\Psi$ & Reference \\
\hline \multirow[t]{6}{*}{$\mathrm{vdW}-\mathrm{DF}$} & $(001)$ & top & -25.5 & 0.295 & 67 & 24 & \\
\hline & & bridge & -22.3 & 0.323 & 72 & 21 & Present \\
\hline & & hollow & -22.1 & 0.365 & 98 & 42 & \\
\hline & $(111)$ & top & -23.3 & 0.303 & 66 & 25 & \\
\hline & & bridge & -19.9 & 0.367 & 111 & 31 & Present \\
\hline & & hollow & -21.2 & 0.368 & 104 & 29 & \\
\hline \multirow[t]{6}{*}{$\mathrm{PBE}$} & $(001)$ & top & -19.3 & 0.268 & 77 & 13 & \\
\hline & & bridge & -18.9 & 0.309 & 91 & 40 & Present \\
\hline & & hollow & -13.0 & 0.366 & 117 & 59 & \\
\hline & $(111)$ & top & -16.5 & 0.276 & 79 & 11 & \\
\hline & & bridge & -11.1 & 0.352 & 115 & 38 & Present \\
\hline & & hollow & -10.8 & 0.370 & 125 & 50 & \\
\hline \multirow[t]{3}{*}{$\mathrm{vdW}-\mathrm{DF}$} & $(001)$ & top & -20.8 & 0.290 & - & - & 16 \\
\hline & & bridge & -17.7 & - & - & - & \\
\hline & & hollow & -15.1 & - & - & - & \\
\hline $\mathrm{vdW}-\mathrm{DF}$ & $(111)$ & top & -18.3 & 0.303 & - & - & 16 \\
\hline $\mathrm{vdW}-\mathrm{DF}$ & $(111)$ & top & -20.0 & - & - & - & 20 \\
\hline optB86b-vdW & $(111)$ & top & -28.9 & 0.269 & - & - & 21 \\
\hline $\mathrm{PBE}$ & $(111)$ & top & -10.6 & 0.279 & - & - & \\
\hline PW91 & $(111)$ & top & -10.8 & 0.285 & - & - & 41 \\
\hline PW91 & $(111)$ & top & -12.5 & 0.302 & 77 & - & 40 \\
\hline
\end{tabular}


hydrogen is pointing toward the surface are less favorable. Although both functionals yield qualitatively similar curves, the interaction using vdW-DF is stronger and the equilibrium distance for both orientations is larger using vdW-DF. We note that multiple variations of vdW functions combined with different exchange functionals have been used to study adsorbate behaviours. A study of small molecules on $\mathrm{Au}(111),{ }^{42}$ found that vdW-DF functionals using either $\mathrm{PBE}$ or revPBE exchange gave adsorption energies in excellent agreement with experimental values, compared to the other vdW functional variations studied. A study of benzene adsorption on gold and platinum surfaces, ${ }^{43}$ found that vdW-DF with revPBE exchange underestimated the experimental adsorption energy on $\mathrm{Pt}(111)$ but vdW-DF with PBE exchange gave reasonable agreement. On $\mathrm{Au}(111)$ vdW-DF with revPBE(PBE) exchange underestimated(overestimated) the experimental adsorption energy. It is unclear if there is a functional that is optimal for all systems, and thus reliable experimental data on the orientation of water near the gold surface are required to determine which functional gives physically correct behaviour.

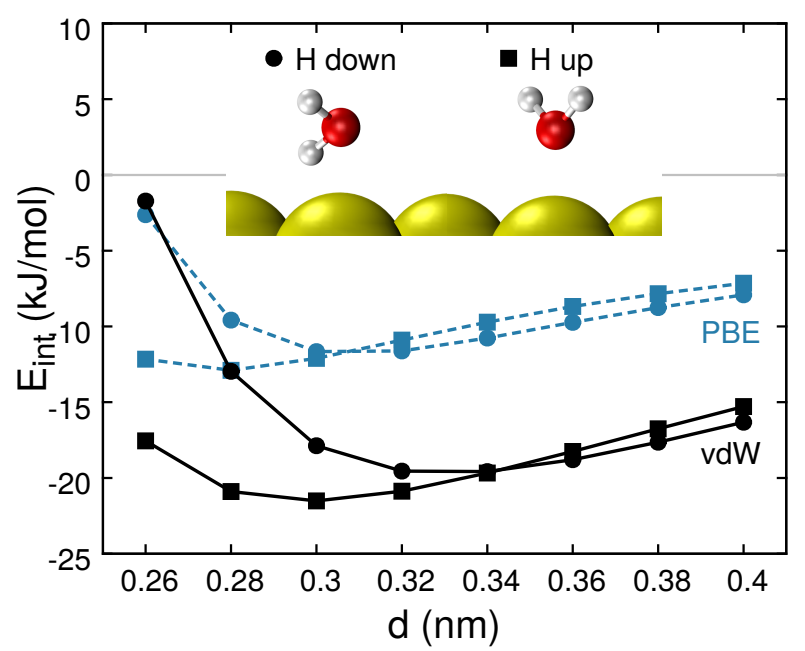

Figure 2: Distance-dependent (Au-O) DFT interaction energies of a water molecule on the $\mathrm{Au}(001)$ surface for two different orientations. Oxygen points towards the surface (squares), hydrogen points towards the surface (circles). vdW-DF results are in black solid lines, PBE results are in blue dashed lines. 


\subsection{Benchmark of existing classical models}

In this subsection, we will assess four parameter sets that were used for studies involving water-gold interactions. We compare the classical force field interaction energies with the DFT interaction energies (vdW-DF) for all water-gold configurations considered in this study. For the present benchmark we tested parameters for models where gold is represented by a single neutral bead per atom and water is represented by a three site water model. We are aware of the limitations and drawbacks of such models and will discuss these issues later. Lennard-Jones (LJ) 12-6 potentials are implemented in most of the current MD software, and three of the tested sets (Sets 1-3) $)^{3,34,44}$ are described by this form of potential. Other

types of pair potentials are available, particularly for heterogeneous systems, and Set $4^{34}$ uses a LJ 9-6 potential.

A generalised LJ m-n potential describes the potential energy, $V_{\mathrm{LJ}}$, between two particles, $i$ and $j$, as a function of their separation distance, $r_{i j}$ :

$$
V_{\mathrm{LJ}}^{m-n}\left(r_{i j}\right)=\epsilon_{i j}\left[\left(\frac{n}{m-n}\right)\left(\frac{r_{m}}{r_{i j}}\right)^{m}-\left(\frac{m}{m-n}\right)\left(\frac{r_{m}}{r_{i j}}\right)^{n}\right]
$$

where $\epsilon_{i j}$ is the energy at the equilibrium distance $r_{m}$. An alternative form of the LJ potential is often used, where the distance corresponding to zero energy, $\sigma$, is used. In these cases the equilibrium distance is related to the zero-energy distance by

$$
r_{m}=\sigma\left(\frac{m}{n}\right)^{(m-n)^{-1}}
$$

For these four force fields the parameters for LJ 12-6 potentials were obtained by the LorentzBerthelot mixing rules (Eq. 3) according to GROMACS and for LJ 9-6 potentials by the 
six power mixing rule (Eq. 4) according to COMPASS:

$$
\begin{aligned}
\epsilon_{i j} & =\sqrt{\epsilon_{i i} \cdot \epsilon_{j j}} & r_{m, i j} & =\frac{r_{m, i i}+r_{m, j j}}{2} \\
\epsilon_{i j} & =2 \cdot \sqrt{\epsilon_{i i} \cdot \epsilon_{j j}}\left(\frac{r_{m, i i}^{3} \cdot r_{m, j j}^{3}}{r_{m, i i}^{6}+r_{m, j j}^{6}}\right) & r_{m, i j} & =\sqrt[6]{\frac{r_{m, i i}^{6}+r_{m, j j}^{6}}{2}}
\end{aligned}
$$

The four sets and their parameters are summarized in Table 2. The water models used for mixing were selected based on the previously-used combinations or recommendations of the authors. Sets 1 and $\mathbf{4}$ are based on $\mathrm{Au}-\mathrm{Au}$ parameters derived in a study by Heinz et al. where potentials were parameterised for a variety of fcc metals to reproduce the bulk properties of the metal, such as surface tension and density. ${ }^{34}$ Set $\mathbf{1}$ is represented by LJ 12-6 potentials in combination with the extended SPC (SPC/E) water model, which does not contain an explicit non-bonded interaction for hydrogen. Set $\mathbf{4}$ is represented by LJ 9-6 potentials in combination with the COMPASS water model which uses this potential type as well and, in this combination, corresponds to the implementation of water-gold interactions in the INTERFACE force field. The COMPASS water model differs not only in the potential type but also contains an explicit interaction for hydrogen.Parameters for Set 2 were taken from a study of a water droplet on a $\mathrm{Au}(111)$ surface $^{3}$ and the gold parameters were mixed with those from SPC/E water. The origin of their gold parameters is unclear. Set 3 parameters are taken from a study of peptides on gold surfaces. ${ }^{44}$ In this study a modified TIP3P model (mTIP3P) was used for water which contains an explicit interaction term for hydrogen. These parameters were obtained by performing MD simulations of a single water droplet on a gold (001) surface at different $\epsilon_{\mathrm{Au}}$ values and a final value for $\epsilon_{\mathrm{Au}}$ was extrapolated to give a contact angle of $0^{\circ}$.

The resulting water-gold potentials differ significantly (Fig. 3), not only in the strength of $\mathrm{Au}-\mathrm{O}$ interactions but also in the nature of the $\mathrm{Au}-\mathrm{H}$ interaction. While Sets 1 and 4 have both moderate $\mathrm{Au}-\mathrm{O}$ interactions, Sets 3 and 4 have an additional $\mathrm{Au}-\mathrm{H}$ term. For Set 4 this $\mathrm{Au}-\mathrm{H}$ term is almost purely repulsive. Set 2 has the strongest $\mathrm{Au}-\mathrm{O}$ interaction 
Table 2: Parameter sets for LJ potentials derived from published $\epsilon_{i}$ and $r_{m, i i}$ values for gold. The $R^{2}$ values show the quality of agreement with DFT interaction energies. $\epsilon_{i j}$ has units $\mathrm{kJ} \mathrm{mol}{ }^{-1}$ and $r_{m}$ is in $\mathrm{nm}$.

\begin{tabular}{lllrrlrr}
\hline Set & Potential & Water model & Ref. & $R^{2}$ & Pair & $\epsilon_{i j}$ & $r_{m}$ \\
\hline 1 & LJ 12-6 & SPC/E & 34 & 0.34 & $\mathrm{Au}-\mathrm{Au}$ & 22.16 & 0.295 \\
& & & & & $\mathrm{Au}-\mathrm{O}$ & 3.80 & 0.325 \\
& & & & $\mathrm{Au}-\mathrm{H}$ & - & - \\
\hline 2 & LJ 12-6 & SPC/E & \multirow{2}{*}{3} & 0.23 & $\mathrm{Au}-\mathrm{Au}$ & 42.57 & 0.296 \\
& & & & & $\mathrm{Au}-\mathrm{O}$ & 5.26 & 0.325 \\
& & & & & $\mathrm{Au}-\mathrm{H}$ & - & - \\
\hline 3 & LJ 12-6 & mTIP3P & \multirow{2}{*}{44} & 0.38 & $\mathrm{Au}-\mathrm{Au}$ & 4.40 & 0.329 \\
& & & & & $\mathrm{Au}-\mathrm{O}$ & 1.67 & 0.341 \\
& & & & & $\mathrm{Au}-\mathrm{H}$ & 0.92 & 0.187 \\
\hline 4 & LJ 9-6 & COMPASS & \multirow{2}{*}{34} & 0.14 & $\mathrm{Au}-\mathrm{Au}$ & 18.10 & 0.300 \\
& & & & & $\mathrm{Au}-\mathrm{O}$ & 3.84 & 0.337 \\
& & & & & $\mathrm{Au}-\mathrm{H}$ & 0.10 & 0.268 \\
\hline
\end{tabular}

due to the high value of $\epsilon_{\mathrm{Au}}=42.57 \mathrm{~kJ} \mathrm{~mol}^{-1}$. Set 3 has has the weakest interaction between water and gold of the sets tested here due to a remarkably low $\epsilon_{\mathrm{Au}}$ of $4.40 \mathrm{~kJ} \mathrm{~mol}^{-1}$ and a $\mathrm{Au}-\mathrm{H}$ term with a weak but significant attractive contribution at very small distance.

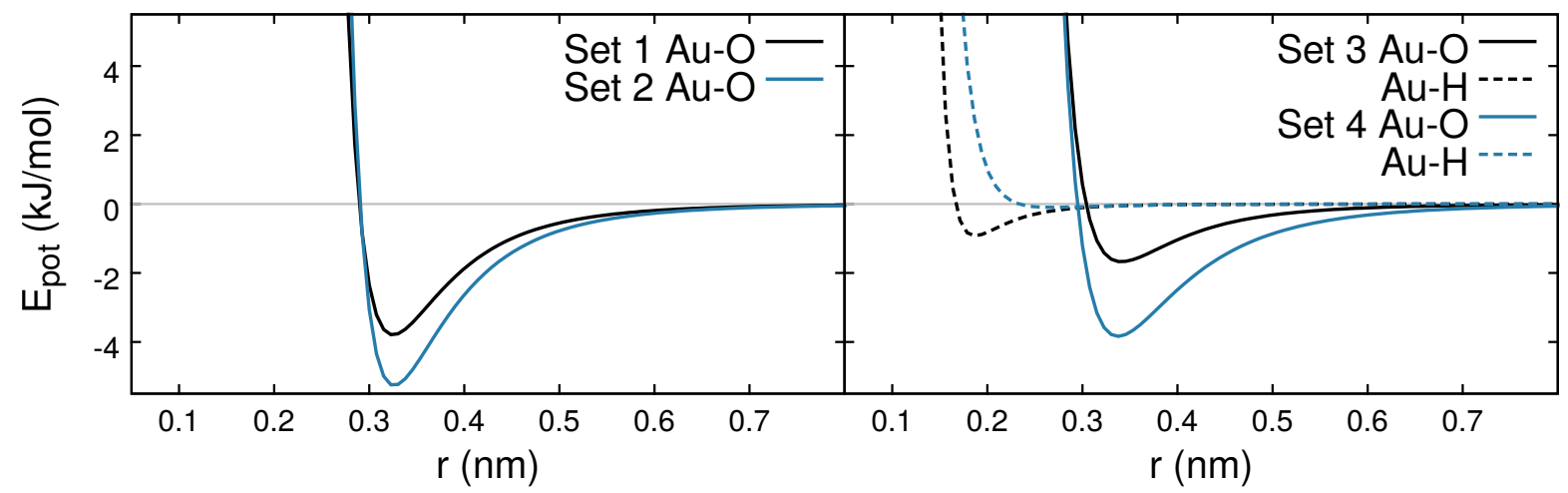

Figure 3: Effective potentials after mixing the gold parameters with non-bonded potentials of water.

The interaction energies for the configurations used in the DFT calculations were used to assess how well the four sets reproduced vdW-DF interaction energies (Fig. 4). For a full view of Figure 4 with high energy configurations please see Figure SI.1 (Supplementary 
Information). The coefficient of determination,

$$
R^{2}=1-\frac{\sum_{i}\left(E_{\mathrm{DFT}, \mathrm{i}}-E_{\mathrm{PEF}, \mathrm{i}}\right)^{2}}{\sum_{i}\left(E_{\mathrm{PEF}, \mathrm{i}}-\bar{E}_{\mathrm{PEF}}\right)^{2}}
$$

was computed as a quality measure between the sets and DFT interaction energies, where for each configuration $i, E_{\mathrm{DFT}, \mathrm{i}}$ is the energy obtained from DFT, $E_{\mathrm{PEF}, \mathrm{i}}$ the energy obtained from the classical model and $\bar{E}_{\mathrm{PEF}}$ is the mean of all energies reproduced by the classical model. An $R^{2}$ value of 1 indicates perfect agreement, and smaller values indicate poorer agreement. Sets 1 and 3 yielded the best results of $R^{2}=0.34$ and $R^{2}=0.38$, respectively. Set 2 had a value of $R^{2}=0.23$ and tended to overestimate the interaction energies, particularly for low energy configurations. The lowest $R^{2}$ value of 0.14 was observed for Set 4 .

The comparison between DFT and classical interaction energies is troubling for two reasons: 1) adsorbed configurations with the lowest DFT energy of -25.5 and $-23.3 \mathrm{~kJ}$ $\mathrm{mol}^{-1}$ on the (001) and (111) gold surface, respectively, were not found to be those with the lowest energy in any of the classical sets, and in particular, Sets 1, 2 and 4 show numerous other configurations to be more favourable than the adsorbed structures. 2) for certain types of configuration a systematic deviation between the interaction energies is observed. We found that configurations dominated by a $\mathrm{Au}-\mathrm{H}$ interaction show the largest deviations between the DFT and classical energies. These results would be expected for Set 1 and 2 where no $\mathrm{Au}-\mathrm{H}$ potential and, consequently, there is no difference in the interaction energies of diverse rotational configurations with the oxygen atom at the same distance in the classical model. This finding is particularly striking considering that Sets 3 and 4 both have a Au$\mathrm{H}$ interaction term. This indicates that the parameterisation choices (LJ pair interactions with a mixing rules approach and a weak hydrogen interaction) of the investigated models limit their ability to reproduce the water-gold interaction energies for the entire conformation space. Furthermore, it should be pointed out that in all four benchmarks, some configurations were predicted to have a negative energy by the DFT calculation while the classical model 
yielded a positive energy and vice versa.

A closer examination of interaction energies of two distinct conformations of a water molecule on the gold (001) surface (one with both $\mathrm{H}$ atoms pointing up and another with one of the $\mathrm{H}$ atoms pointing down towards the surface) gives further insight on the reason for the observed deviations between DFT and classical energies (Fig. 5). As already shown, DFT calculations predict a similar energy for both orientations of water in the range $3.4 \mathrm{~nm}-$ $4.0 \mathrm{~nm}$. This behaviour changes significantly for closer distances and reveals a less attractive energy for the hydrogen dominated orientation. The four forcefields show different capabilities of reproducing DFT interaction energies for these configurations but none of them is able to distinguish energetically between the two orientations. Even though Sets 3 and 4 have interaction potentials for hydrogen it appears that they do not contribute significantly in the range investigated here and the most obvious reason is the low $r_{m, \mathrm{H}}$ values in both mTIP3P and COMPASS water models which result in very short range potentials between gold and hydrogen (Fig. 3). In view of minimum energy configurations found by DFT where water is adsorbed almost flat on top of the gold surface and hydrogen has a slightly larger distance to the surface than oxygen $r_{m, \mathrm{Au}-\mathrm{H}}$ should be increased to reproduce DFT results.

\subsection{Optimization of Potential Energy Functions}

Our next aim is to use interaction energies from DFT calculations to find improved parameters for the non-bonded potentials by a fitting procedure. Structures from DFT calculations together with corresponding calculated interaction energies were used as input for the GULP package $^{28}$ to optimize input potential parameters. We have taken two different approaches for the optimization procedure:

1. Optimize only the gold parameters, $\epsilon_{\mathrm{Au}}$ and $r_{m, \mathrm{Au}}$, which were mixed during the fitting procedure with parameters for water. This was performed for both types of water models (with and without a hydrogen interaction potential) and two potential forms (LJ 12-6 and LJ 9-6). 

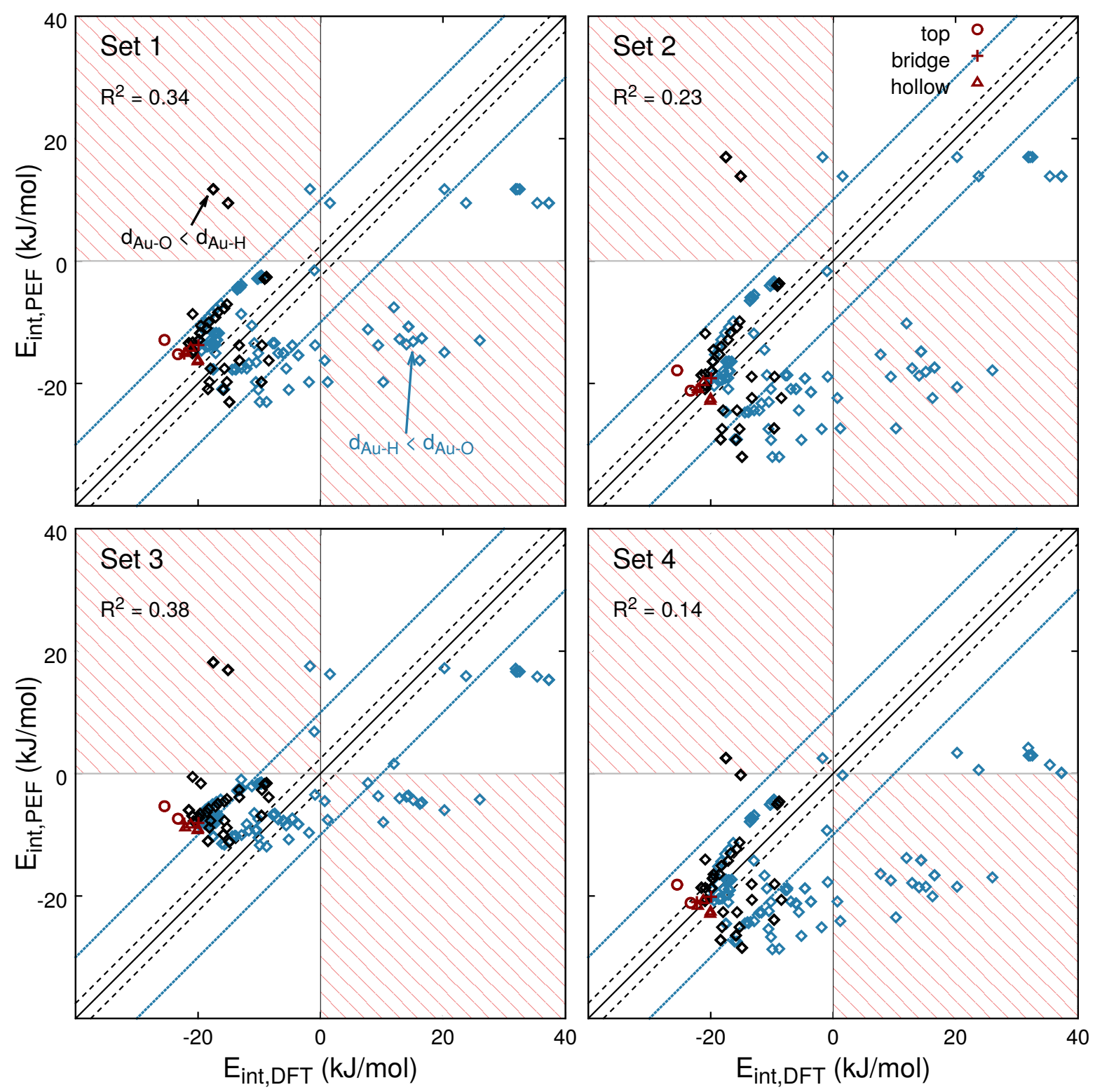

Figure 4: Comparison of interaction energies from vdW-DFT and classical calculations (Set 1-4). Configurations where oxygen is closer to the surface (black) and where the hydrogen is closer to the surface (blue). Deviation of $10 \pm \mathrm{kJ} \mathrm{mol}^{-1}$ from linear correlation (blue solid lines) and of $\pm 2.5 \mathrm{~kJ} \mathrm{~mol}^{-1}\left(k_{B} \cdot 300 \mathrm{~K}\right.$, black dashed lines). Areas where energy is positive in one model but negative in the second one are shaded red. Adsorbed configurations obtained by calculations where water atoms were allowed to move (red symbols, key in upper right graph). 


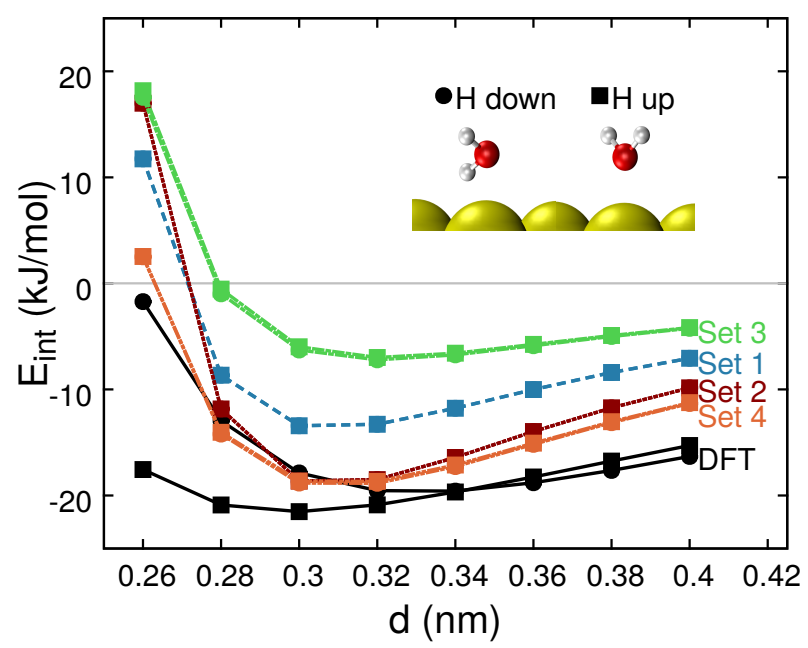

Figure 5: Comparison of interaction energies from DFT and classical calculations for two distinct conformations of a water molecule over a range of distances to the gold (001) surface. While Set $\mathbf{2}$ and $\mathbf{4}$ are reproducing the energies fairly well at the equilibrium distance none of the classical models are able to reproduce the deviation between conformations where the oxygen is pointing towards the surface (squares) and those where hydrogen is closer to the surface (circles). Circles are on top of squares for classical models.

2. Optimize both the $\mathrm{Au}-\mathrm{O}$ and $\mathrm{Au}-\mathrm{H} \mathrm{LJ}$ interactions for different potential forms without using mixing rules.

For the two cases we present the results of optimization for different potential types against interaction energies as well as adsorption energies from DFT calculations. Subsequently, we will show that the optimization is transferable regarding the gold geometry and selfconsistency for the set of DFT reference calculations performed in this study. Finally, we discuss the limitations of these approaches.

\subsubsection{Optimization of Gold LJ Potential Parameters}

In most cases force field optimization involves the variation of as few parameters as possible to prevent drastic unforeseen changes in properties other than those which were optimised. Furthermore, to keep the number of force field parameters small, specific interaction potentials are defined for distinct atomic types and mixed to obtain potentials for all pairwise combinations of a system. The most general and transferable solution which is usually used 
in force fields for biomolecules, would be a parameter set for gold which can be mixed to obtain heterogeneous interaction potentials for all kinds of atomic types. This approach would have the advantage that the optimized $\epsilon_{\mathrm{Au}}$ and $r_{m, \mathrm{Au}}$ values could be used in combination with whole force fields. This approach also has its drawbacks. For example, if parameters for gold are optimised to reproduce interactions with other atoms or molecules these gold parameters will not accurately reproduce the bulk properties of metallic gold. The ability of LJ potentials to reproduce all relevant properties of gold, such as surface reconstructions, ${ }^{16}$ is questionable but outside the scope of this study. Nevertheless, in this section we follow this approach to test its ability to reproduce DFT results.

The most commonly used water models (SPC/E, TIP3P) do not include an explicit non-bonded potential for hydrogen, so hydrogen interacts only by electrostatic interactions with other charged particles but not with neutral gold atoms. It was already shown in the previous section that the DFT interaction energy of a water molecule on gold is highly dependent on the orientation and that existing parameter sets are not capable of reproducing the orientational dependence. Optimization of the Au interaction potential via parameters $\epsilon_{\mathrm{Au}}$ and $r_{m, \mathrm{Au}}$, resulted in improved but still poor $R^{2}$ values of 0.48 and 0.55 for LJ $12-6$ and 9-6 potential types, respectively (Fig. SI.2).

To show if it is possible to obtain a parameter set for gold which can be combined with existing force fields for a detailed description of the conformational structure of water near to a gold surface we selected two water models, mTIP3P and COMPASS, with an explicit non-bonded term for hydrogen. Parameters for gold $\left(\epsilon_{\mathrm{Au}}\right.$ and $\left.r_{m, \mathrm{Au}}\right)$ were mixed during the fitting procedure with the corresponding water model to obtain $\mathrm{Au}-\mathrm{O}$ and $\mathrm{Au}-\mathrm{H}$ potentials. We obtained a minor improvement using an LJ 9-6 potential, which yielded $R^{2}=0.62$ but an LJ 12-6 potential yielded a worse result of $R^{2}=0.45$ than those force fields that omitted the $\mathrm{H}$ interaction (Fig. SI.2).

We observed that optimization of force fields without a gold-hydrogen interaction or force fields with intermolecular interactions obtained by mixing rules gave unsatisfactory 
results. We conclude that gold-hydrogen parameters, particularly $r_{m}$, must be allowed to vary significantly. Therefore, in the following subsection, we will focus on fitting the goldoxygen and gold-hydrogen parameters without the use of mixing rules.

\subsubsection{Optimization of Interface Potentials Without Mixing Rules}

We decided to extend this study by including three additional potential types to investigate the influence of the potential form on the optimization result in more detail. The LJ 10-4 potential is described by the generalised LJ potential (Eq. 2). Even though this potential type was derived for unstructured surfaces we will show that it performs better than LJ 12-6 and 9-6 in describing the water-gold interaction. The Buckingham (Eq. 5) and Morse ${ }^{45}$ (Eq. 6) potentials describe the potential energy between two particles, $i$ and $j$, as a function of the distance between them, $r_{i j}$ :

$$
\begin{aligned}
V_{\text {Buck }}\left(r_{i j}\right) & =A e^{-\frac{r_{i j}}{\rho}}-C r_{i j}^{-6} \\
V_{\text {Morse }}\left(r_{i j}\right) & =D\left[\left[1-e^{-\alpha\left(r_{i j}-r_{m}\right)}\right]^{2}-1\right]
\end{aligned}
$$

Each potential has three adjustable parameters: $A, \rho$ and $C$ for Buckingham and $D, \alpha$ and $r_{m}$ for Morse. The additional parameter should have beneficial effects on the optimization outcome, and was found to give better fit for benzene on gold. ${ }^{19}$ While repulsion only is described by an exponential function in Buckingham potentials both the attraction and repulsion are exponential in Morse potentials. Therefore, we are able to investigate the ability of exponential terms to describe water-gold interactions.

Optimization was performed for five different potential types (Fits 1-5). The outcome of the fitting was independent from the starting parameters for distinct potential types provided that they were physically realistic. For each potential type we found parameters (Tab. 3) that give better results in reproduction of DFT interaction energies than the existing sets in Section 3.2 (Fig. 7). 
Table 3: Parameters for five different potential types obtained by optimization against DFT energies. $\epsilon_{i j}, A$ and $D$ have units $\mathrm{kJ} \mathrm{mol}^{-1}, r_{m}$ and $\rho$ are in $\mathrm{nm}, \alpha$ is in $\mathrm{nm}^{-1}$, and $C$ is in $\mathrm{kJ} \mathrm{mol}^{-1} \mathrm{~nm}^{-6}$.

\begin{tabular}{|c|c|c|c|c|c|c|}
\hline Fit & Potential & $R^{2}$ & Pair & \multicolumn{3}{|c|}{ Parameters $^{b}$} \\
\hline & & & & $\epsilon_{i j}$ & $r_{m}$ & \\
\hline \multirow[t]{2}{*}{1} & LJ 12-6 & 0.74 & $\mathrm{Au}-\mathrm{O}$ & 3.61 & 0.318 & \\
\hline & & & $\mathrm{Au}-\mathrm{H}$ & 0.01 & 0.395 & \\
\hline \multirow[t]{2}{*}{2} & LJ 9-6 & 0.84 & $\mathrm{Au}-\mathrm{O}$ & 2.91 & 0.336 & \\
\hline & & & $\mathrm{Au}-\mathrm{H}$ & 0.05 & 0.375 & \\
\hline \multirow[t]{3}{*}{3} & LJ 10-4 & 0.89 & $\mathrm{Au}-\mathrm{O}$ & 1.41 & 0.366 & \\
\hline & & & $\mathrm{Au}-\mathrm{H}$ & 0.02 & 0.415 & \\
\hline & & & & $A$ & $\rho$ & $C$ \\
\hline \multirow[t]{3}{*}{4} & Buckingham & 0.96 & $\mathrm{Au}-\mathrm{O}$ & 45636 & 0.043 & 0.027 \\
\hline & & & $\mathrm{Au}-\mathrm{H}$ & 13218 & 0.033 & 0.000 \\
\hline & & & & $D$ & $\alpha$ & $r_{m}$ \\
\hline \multirow[t]{2}{*}{5} & Morse & 0.97 & $\mathrm{Au}-\mathrm{O}$ & 1.86 & 9.05 & 0.420 \\
\hline & & & $\mathrm{Au}-\mathrm{H}$ & 0.08 & 14.1 & 0.414 \\
\hline
\end{tabular}

The worst potential fit was the most commonly used LJ 12-6 potential, which gave an $R^{2}$ value of 0.74 (Fit 1). The agreement between DFT and classical interaction energies is better for LJ 9-6 (Fit 2) and 10-4 (Fit 3) potentials. However, a significant improvement was observed for Buckingham (Fit 4) and Morse (Fit 5) potentials with $R^{2}$ values of 0.96 and 0.97 , respectively. It seems that nature of water-gold interactions are described best by potentials where repulsion has an exponential shape since there is only a minor difference between the Morse and Buckingham fits. This agrees with a previous study of benzene on $\mathrm{Au}(111)$, which also showed that Morse potentials reproduced DFT interaction energies better than LJ potentials. ${ }^{18}$

For all fits, the $\mathrm{Au}-\mathrm{H}$ interaction is almost purely repulsive for distances below $\approx 0.3 \mathrm{~nm}$ (Fig. 6). The optimised Morse and Buckingham potentials have a significantly softer shape than the LJ potentials, and the optimized Buckingham potential has no $\mathrm{Au}-\mathrm{H}$ attraction $\left(C=0.000 \mathrm{~kJ} \mathrm{~mol}^{-1} \mathrm{~nm}^{6}\right)$. Indeed this emphasises the problem that in all the other potentials used in this study, including Morse, there is only one energy parameter which adjusts both the attractive and repulsive interaction terms of the potential simultaneously. For these 


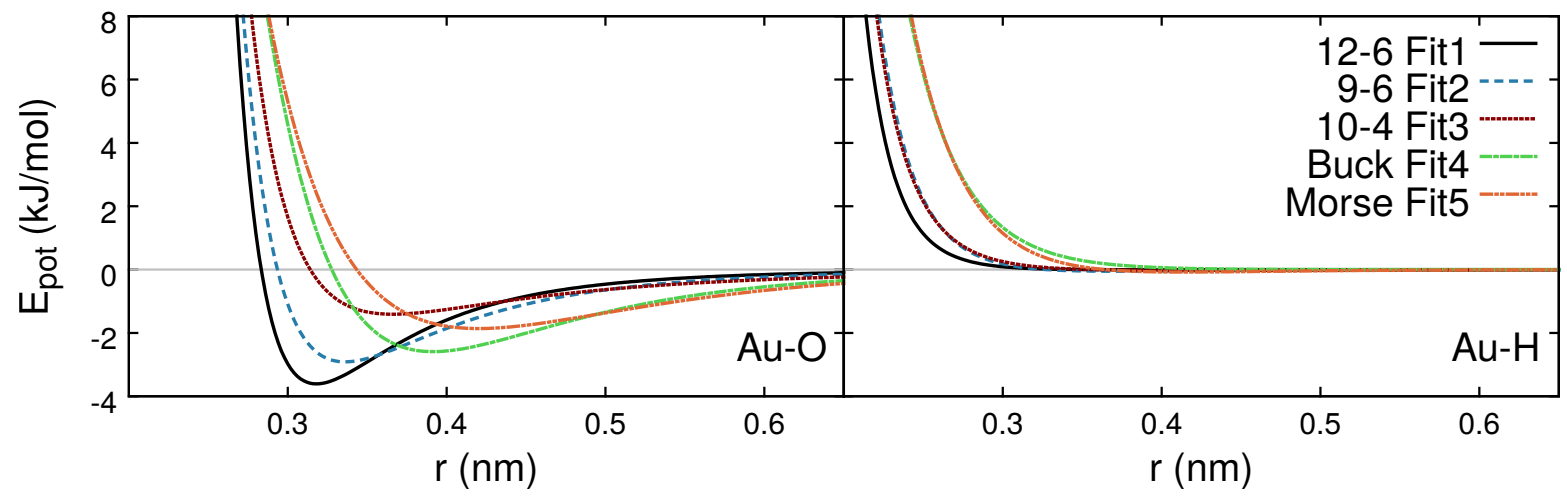

Figure 6: Interaction potentials obtained by optimization against DFT energies. Left: goldoxygen; right: gold-hydrogen.

potentials the optimization resulted in a very weakly attractive $\mathrm{Au}-\mathrm{H}$ interaction with a relatively large equilibrium distance (compared to the $\mathrm{Au}-\mathrm{O}$ equilibrium distance). Compared to the hydrogen atom's van der Waals radius this finding seems unphysical but a repulsive interaction of this form is required to reproduce the adsorbed conformation of water correctly. Optimised $\mathrm{Au}-\mathrm{O}$ potentials, on the other hand, differ significantly in equilibrium energy and distance. In general, the equilibrium distance is larger and interaction is weaker than in potentials obtained from literature for fits with good agreement.

Linear agreement between interaction and adsorption energies from DFT calculations and classical model is significantly increased for Buckingham (Fit 4) and Morse (Fit 5) potentials and all energies are in the range of $\pm 10 \mathrm{~kJ} \mathrm{~mol}^{-1}$ (Fig. 7). However, it was not possible to obtain parameters for gold-water pair potentials where the lowest energy configuration of a water molecule on top of a gold atom (red circles in Figure 7) is also the lowest energy configuration in the classical model. Indeed, the preferred conformation for potentials from Fits 4 and 5 is the adsorbed structure on the hollow site (red triangle) instead of the top site (red circle). This difference in site preference is because a system of pair potentials tends to maximise the number of neighbour interactions resulting in the hollow site, whereas DFT calculations describe a complex electronic interaction, which is often the top site for water molecules on metal surfaces. ${ }^{46}$

In an additional step we also used Boltzmann weighting during optimization (Fig. SI.4). 
Although low energy conformations showed better agreement for Buckingham and Morse potentials compared to a non-weighted fitting, the optimization outcome was worse for LJ potentials, even for low energy conformations. Furthermore, for all potentials, regardless of the weighting, the top adsorption site is not the lowest energy conformation.

\subsubsection{Transferability}

Our aim is to obtain force field parameters which can be used in a transferable way for

all gold surfaces. Such a force field would have the advantage that it can be used for simulations containing various gold surfaces in a single simulation box, such as a system with gold nanoparticles. Therefore, optimisation of parameters was performed using interaction energies calculated either on the (001) or the (111) surface.

We observed that fitting to interaction energies from one surface gives a good fit for the other surface. Force field parameters obtained from fitting to the entire set of 174 configurations lie in between the parameters obtained from separate fits (Fig. 8). Furthermore, Morse potentials from fitting against these three different data sets $(\mathrm{Au}(001), \mathrm{Au}(111)$ and both) are remarkably similar and indicate that this potential type is particularly suitable for a transferable force field.

\subsection{Simulations}

To compare the performance of the nine force fields, we investigate the structure and dynamics of a water layer between two parallel gold surfaces using classical MD simulations. The results of the MD simulations are compared with ab initio MD (AIMD) results in the literature. The nine force fields are the four taken from literature (Sets 1-4, Table 2) and the five optimized force fields (Fits 1-5, Table 3). Snapshots of the water film confined between two $\mathrm{Au}(001)$ and two $\mathrm{Au}(111)$ surfaces are shown in Figure 9. The first layer of water can be clearly seen at the surface. Water density profiles as a function of the distance from the gold surface show the formation of two well-defined layers with significantly higher 


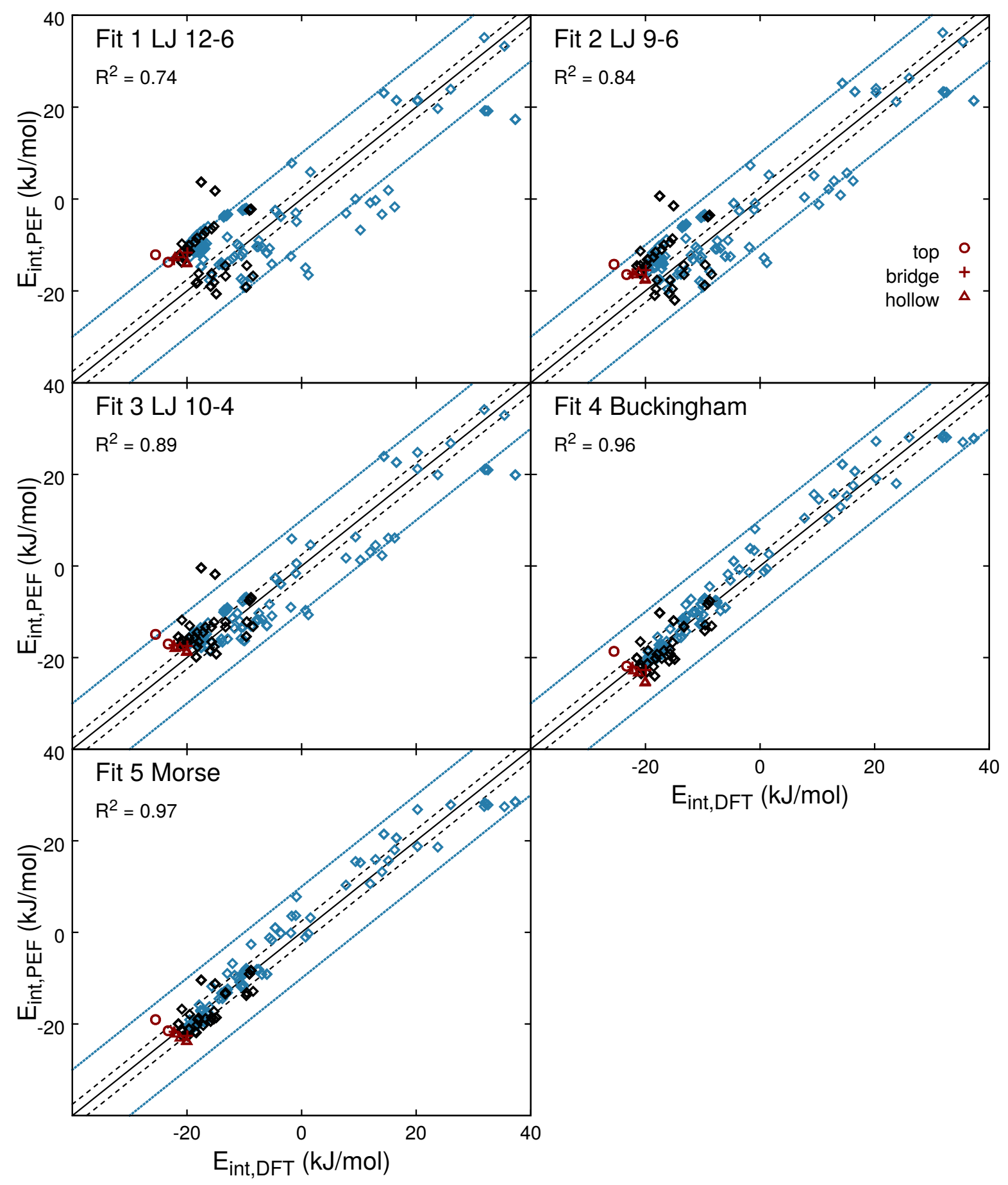

Figure 7: Comparison of interaction energies from DFT and classical calculations (Fit 1-5). Configurations where oxygen is closer to the surface (black) and where the hydrogen is closer to the surface (blue). Deviation of $\pm 10 \mathrm{~kJ} \mathrm{~mol}^{-1}$ from linear correlation (blue solid lines) and of $\pm 2.5 \mathrm{~kJ} \mathrm{~mol}^{-1}\left(k_{B} \cdot 300 \mathrm{~K}\right.$, black dashed lines). Adsorbed configurations obtained by calculations where water atoms were allowed to move (red symbols, key in upper right graph). 


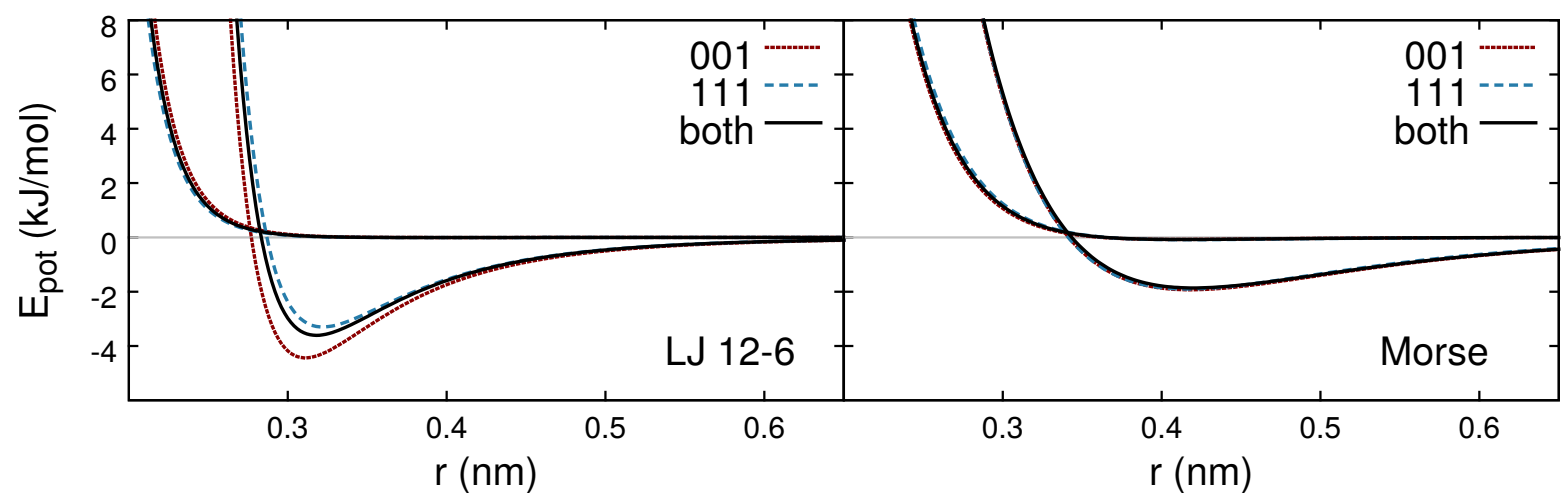

Figure 8: Optimised potentials LJ 12-6 (left) and Morse (right) based on DFT energies from the 001 (red solid), 111 (blue dashed) and both surfaces (black solid).

density than bulk water (Fig. 10). The optimized force fields yielded lower first layer maximal densities, which is similar to the densities from the GolP force field results published by Wright et al.. ${ }^{16}$ Density maxima are shifted to larger distances for fits which had higher $R^{2}$ values. The normalised water density from AIMD simulations on the $\mathrm{Au}(111)$ surface $^{21}$ has a distinctly different shape with two separate peaks appearing for the first water layer at the gold surface. This feature was not observed in MD simulations so far and it is unclear whether this is reproducible using classical pair potential models. AIMD simulations of water on $\mathrm{Au}(111)$ by Pedroza et al. found no evidence of a double oxygen peak in the density profile around 3-4 $\AA{ }^{12}$ Another AIMD study by Cicero et al. ${ }^{11}$ observed a shoulder in the density profile next to the major maxima but the density profile was shown for water molecules only and not oxygen and hydrogen separately.

The first water layer (ranging between 0.2 and $0.45 \mathrm{~nm}$ from the surface) exhibits inplane structure due the gold surface Figure SI.5 (Supplementary Information). Although, this pattern is slightly more pronounced for force fields with deeper potentials, the differences for the various potential forms tested here were not really significant. In all cases the density maxima of water was found to be above the hollow sites of the surface. This is not surprising since this was the lowest energy conformation predicted by all of the classical models in Figure 6. This is one of the limitations of the classical force field used in this study which solely rely on pair potentials for the interaction between the gold atoms and water, as will 

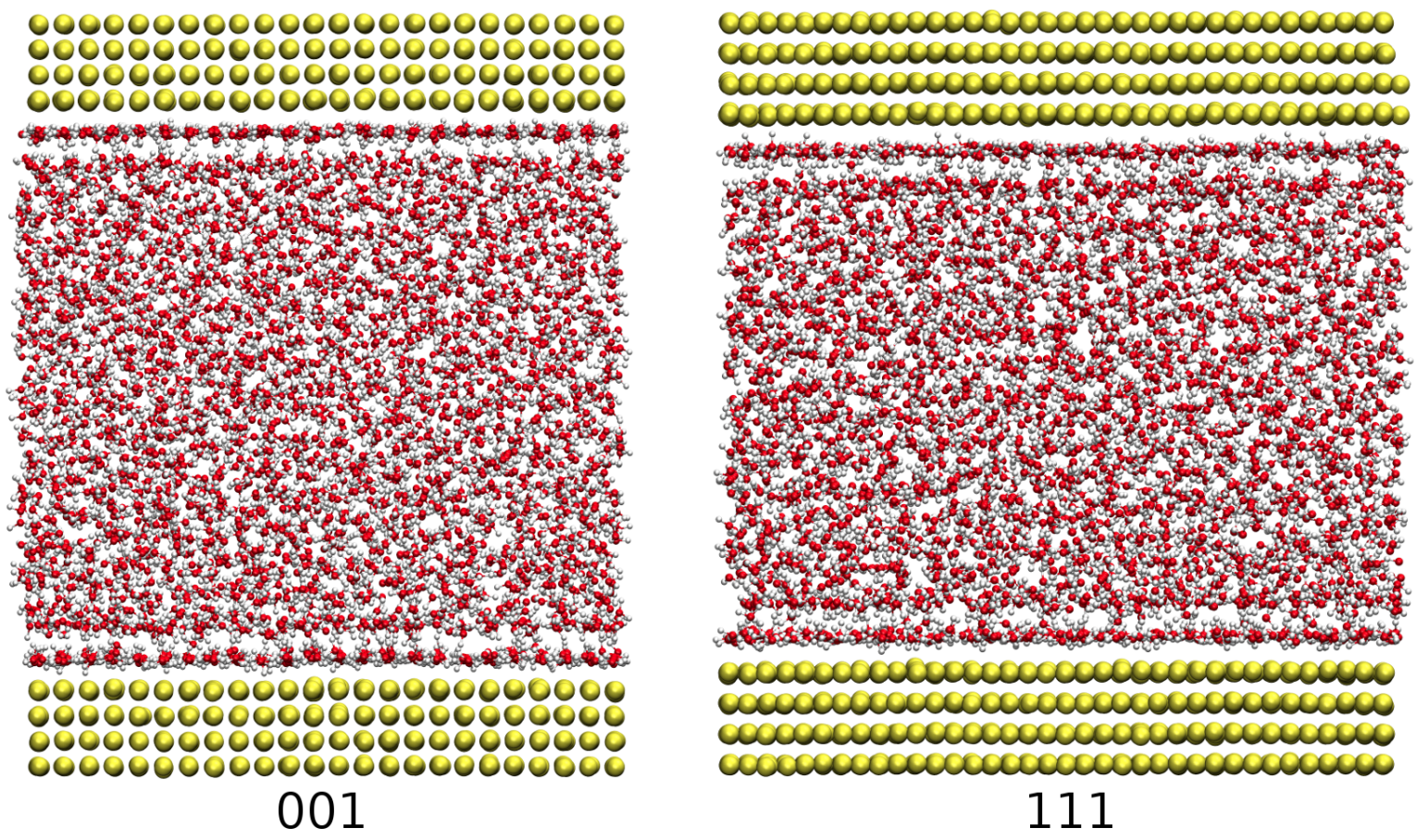

Figure 9: Snapshot of water film confined between two (001) gold surfaces (left) and two (111) gold surfaces (right).

be discussed in more detail later.

The orientation of water molecules near the gold surface may affect the nature of waterwater interactions but also may affect water-solute interactions in simulations of more complex systems such as proteins in the presence of gold. Distributions of the angles $\Phi$ and $\Psi$, according to Section 3.1, for the first layer $(0.2-0.45 \mathrm{~nm})$ obtained from simulations with parameters from Fit 5 are shown in Figure 11. On both surfaces there were two differently populated states indicating two types of adsorbed water molecules next to the gold interface. Most of the water molecules are oriented almost flat but with the dipole oriented slightly towards the surface $\left(80^{\circ}<\Phi<120^{\circ}\right)$ and the molecule plane slightly tilted so that one hydrogen atom is oriented towards the surface $\left(3^{\circ}<\Psi<30^{\circ}\right)$. Significantly fewer water molecules are oriented in a way that the dipole is turned towards the second water layer $\left(40^{\circ}<\Phi<70^{\circ}\right)$ with a broad distribution for $\Psi$ with a maximum around $85^{\circ}$. Interestingly, this is in good agreement with results from first principle simulations performed by Nadler 

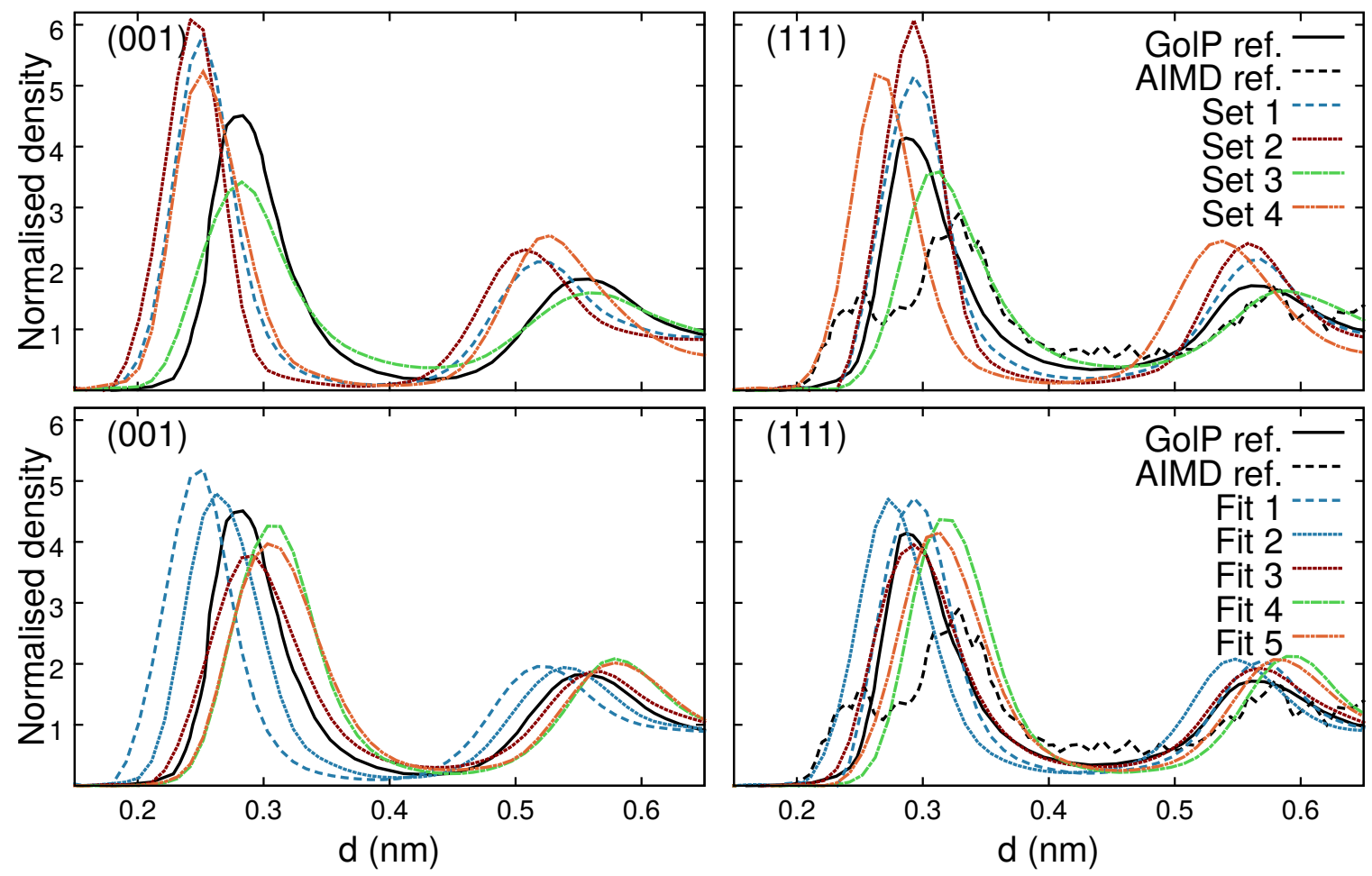

Figure 10: Normalized water density in the $z$-direction. Force fields from literature (Set 1-4) upper row, optimized force fields (Fits 1-5) lower row. For comparison: density from ref. 16 (Fig. 8), GolP FF (black solid line); Oxygen density on the Au(111) surface found by AIMD simulations in ref. 21 (Fig. 7) (back dashed line). 
and Sanz where this feature appeared when functionals with vdW exchange were used. ${ }^{21}$

These angle distributions are comparable for all simulations performed here (Fig. SI.6). However, for the optimised force fields we observe that the two types of adsorbed water molecule are also at different distances from the surface. While there is apparently no distance variation between these two types in simulations with force fields from literature, we observe distribution maxima at $0.30 \mathrm{~nm}$ and $0.32 \mathrm{~nm}$ for the simulation with Morse potentials on the $\mathrm{Au}(001)$ surface. This might indicate the formation a more pronounced water structure close to the gold interface.

To analyse the influence of force fields on dynamic properties, such as dipole autocorrelation and diffusion of water molecules inside the two layers, we searched for trajectories of water molecules that spent at least 200 ps inside a certain layer without transition to a different layer. The ranges were $0.2-0.45 \mathrm{~nm}$ and $0.45-0.7 \mathrm{~nm}$ for the first and second layers, respectively. These single molecule trajectories were extracted, analysed and averaged (Tab. 4). Since the diffusion of water was particularly high in Set 3, there were no molecules in the second layer which remained in a layer for at least 200 ps. The diffusion constant and dipole autocorrelation time of water are highly influenced by the water model, which makes it hard to compare the results of Set 3 and 4, which use mTIP3P and COMPASS water models, respectively, with the other force fields that use the SPC/E water model.

For the three water models used in this study, the diffusion constants of bulk water were determined to be $2.76 \times 10^{-9}, 5.70 \times 10^{-9}$ and $7.32 \times 10^{-9} \mathrm{~m}^{2} \mathrm{~s}^{-1}$ for SPC/E, mTIP3P and COMPASS, respectively. These values are in good agreement with $2.79 \times 10^{-9}$ and $5.88 \times 10^{-9} \mathrm{~m}^{2} \mathrm{~s}^{-1}$ for $\mathrm{SPC} / \mathrm{E}$ and $\mathrm{mTIP} 3 \mathrm{P}$ water, respectively, which were determined by Mark and Nilsson. ${ }^{47}$ Although all water models tend to overestimate diffusion rates compared to the experimental result ${ }^{48}$ of $2.3 \times 10^{-9} \mathrm{~m}^{2} \mathrm{~s}^{-1}$ the models will be used as the references for this study.

We observed that the nature and strength of the gold-water interaction has a minor effect on the diffusion inside the second layer, which is always reduced by $50-70 \%$ compared 


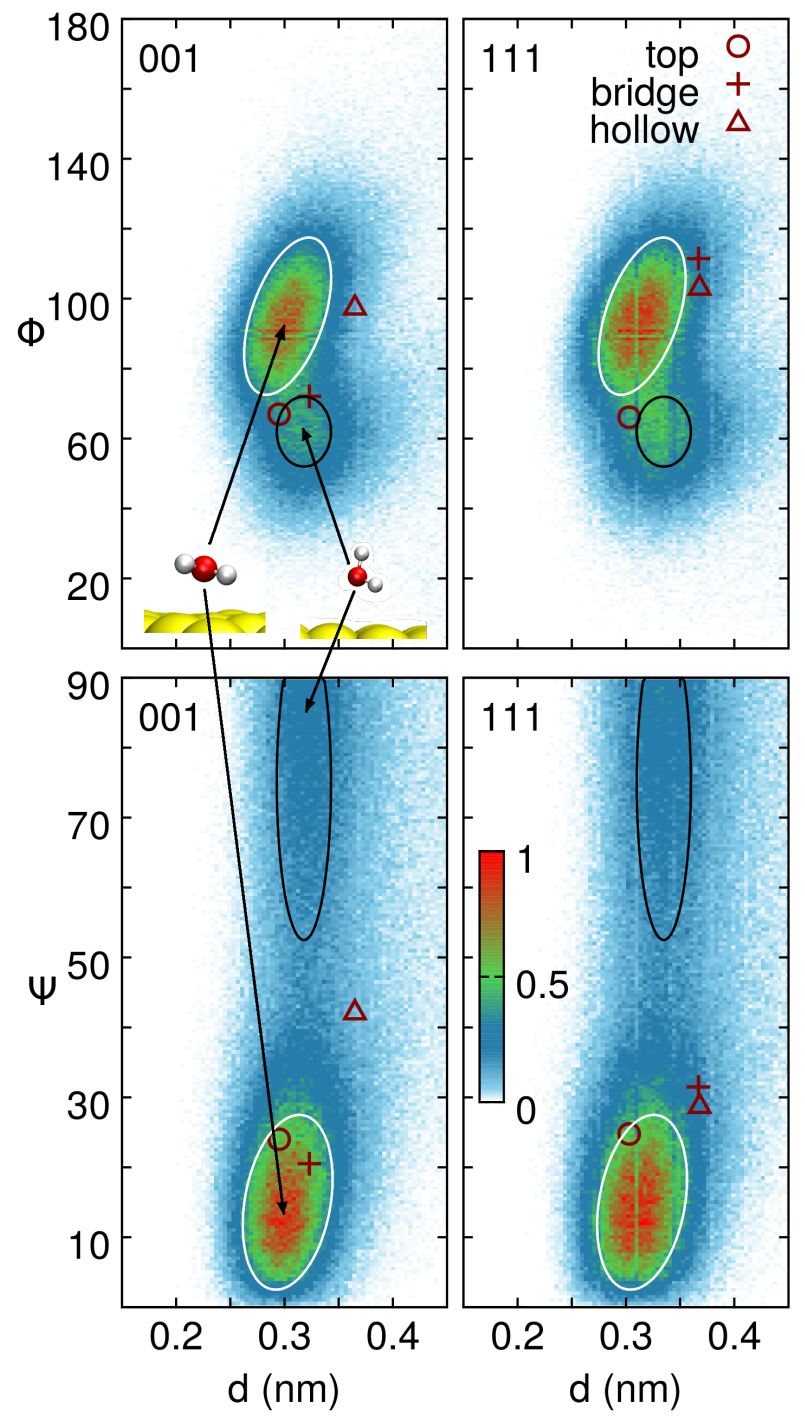

Figure 11: Orientation of water at the gold interface. Distribution of water as a function of angles $\Phi$ and $\Psi$ as defined in Figure 1 and distances to the surfaces $\mathrm{Au}(001)$ and $\mathrm{Au}(111)$. Data was obtained from simulations with parameters from Fit 5 (Morse potentials). First layer between $0.20-0.45 \mathrm{~nm}$ is shown. Two different populated states are marked by white and black ellipses and corresponding orientations of water as insets. Adsorbed configurations obtained by vdW-DF calculations are shown as red symbols. Adsorption site according to key. 
to bulk water. On the other hand, diffusion inside the first layer is highly dependent on the force field. In particular, on the gold (001) surface we observed that LJ potential force fields result in a first water layer that is almost frozen with diffusion constants $<1 \%$ of bulk water. For softer PEFs such as the Morse potential we found the diffusion to be significantly faster with a diffusion constant of $11 \%$ and $21 \%$ of the bulk water reference values on the $\mathrm{Au}(001)$ and $\mathrm{Au}(111)$ surfaces, respectively.

The water dipole autocorrelation time $\tau_{\text {dip }}$ for bulk SPC/E water was found to be $4.5 \mathrm{ps,}$ which is slightly lower than the value of 4.9 ps determined by Kumar et al.. ${ }^{49}$ For the mTIP3P and COMPASS water models we determined $\tau_{\text {dip }}$ to be $2.2 \mathrm{ps}$ and $1.3 \mathrm{ps}$, respectively. As one would expect the rotation of water molecules is significantly hindered inside the first water layer but only slightly affected $\left(\sim 1.5 \tau_{\text {dip }}\right.$ of bulk) inside the second layer. Fits 1 and 2 gave the slowest reorientation of the water dipole near the $\mathrm{Au}(001)$ surface and Fit 3 gave the fastest reorientation. Surprisingly, the dipole autocorrelation time is increased for optimised force fields compared to the sets from literature. Overall, this means that the translation of water molecules is increased and reorientation is decreased by our fitted potentials compared to literature sets.

Although at present we are not able to judge which of the results are physically correct, we have shown that the dynamic properties of water at the gold interface are highly sensitive to even minor deviations in the force field used for the MD simulation. Therefore, the choice of force field might have an enormous impact on related properties, such as interface-induced folding and unfolding of proteins. This emphasises the need for additional experimental data and further effort on the parametrisation and validation of a realistic force field for water-gold interactions. 
Table 4: Dynamical properties of water at gold interfaces and in bulk. Diffusion constants $D$ are in $10^{-9} \mathrm{~m}^{2} \mathrm{~s}^{-1}$ and water dipole autocorrelation times $\tau_{\text {dip }}$ are in ps.

\begin{tabular}{|c|c|c|c|c|c|c|c|c|c|}
\hline & \multirow[b]{3}{*}{ water model } & \multicolumn{4}{|c|}{$D$} & \multicolumn{4}{|c|}{$\tau_{\text {dip }}$} \\
\hline & & \multicolumn{2}{|c|}{ 1st layer } & \multicolumn{2}{|c|}{ 2nd layer } & \multicolumn{2}{|c|}{ 1st layer } & \multicolumn{2}{|c|}{ 2nd layes } \\
\hline & & 001 & 111 & 001 & 111 & 001 & 111 & 001 & 111 \\
\hline Set 1 & $\mathrm{SPC} / \mathrm{E}$ & 0.00 & 0.35 & 1.25 & 1.30 & 38.3 & 20.9 & 6.2 & 6.2 \\
\hline Set 2 & $\mathrm{SPC} / \mathrm{E}$ & 0.00 & 0.13 & 1.15 & 1.17 & 30.6 & 29.6 & 6.2 & 6.5 \\
\hline Set 3 & mTIP3P & 0.94 & 2.11 & - & - & 7.0 & 6.0 & - & - \\
\hline Set 4 & COMPASS & 0.04 & 0.54 & 2.92 & 2.14 & 9.9 & 6.6 & 2.0 & 2.9 \\
\hline Fit 1 & $\mathrm{SPC} / \mathrm{E}$ & 0.00 & 0.33 & 1.07 & 1.11 & 61.4 & 38.1 & 8.3 & 8.9 \\
\hline Fit 2 & $\mathrm{SPC} / \mathrm{E}$ & 0.01 & 0.35 & 1.04 & 1.18 & 60.0 & 38.9 & 8.4 & 7.9 \\
\hline Fit 3 & $\mathrm{SPC} / \mathrm{E}$ & 0.25 & 0.61 & 1.11 & 1.31 & 30.9 & 26.8 & 7.2 & 7.2 \\
\hline Fit 4 & $\mathrm{SPC} / \mathrm{E}$ & 0.13 & 0.49 & 1.07 & 1.21 & 47.6 & 38.9 & 7.6 & 7.8 \\
\hline \multirow[t]{3}{*}{ Fit 5} & $\mathrm{SPC} / \mathrm{E}$ & 0.31 & 0.58 & 1.18 & 1.34 & 36.7 & 35.6 & 7.4 & 7.4 \\
\hline & & \multicolumn{8}{|c|}{ bulk water } \\
\hline & & \multicolumn{4}{|c|}{$D$} & \multicolumn{4}{|c|}{$\tau_{\text {dip }}$} \\
\hline Present & $\mathrm{SPC} / \mathrm{E}$ & \multicolumn{4}{|c|}{2.76} & \multicolumn{4}{|c|}{4.5} \\
\hline Present & mTIP3P & \multicolumn{4}{|c|}{5.70} & \multicolumn{4}{|c|}{2.2} \\
\hline Present & COMPASS & \multicolumn{4}{|c|}{7.32} & \multicolumn{4}{|c|}{1.3} \\
\hline 47 & $\mathrm{SPC} / \mathrm{E}$ & \multicolumn{4}{|c|}{2.79} & \multicolumn{4}{|c|}{-} \\
\hline 49 & $\mathrm{SPC} / \mathrm{E}$ & \multicolumn{4}{|c|}{-} & \multicolumn{4}{|c|}{4.9} \\
\hline 47 & mTIP3P & \multicolumn{4}{|c|}{5.88} & \multicolumn{4}{|c|}{-} \\
\hline 48 & exp. & \multicolumn{4}{|c|}{2.3} & \multicolumn{4}{|c|}{ - } \\
\hline
\end{tabular}




\section{Discussion and Conclusion}

This study assessed the performance of four published force fields for a water-gold interface and developed five optimised force fields using vdW-DFT calculations as a benchmark. We used vdW-DF calculations to obtain the interaction energy of a water molecule with a gold surface in a variety of configurations. We used 86 configurations for the $\mathrm{Au}(111)$ surface and 88 for the $\mathrm{Au}(001)$ surface. We found that LJ 12-6 and LJ 9-6 force fields were not able to reproduce the energy landscape for the wide conformational space used for benchmarking. The major reasons for the poor performance were a) no interaction term for hydrogen was present or b) the potentials for hydrogen were too short ranged. Furthermore, it was not possible to obtain gold parameters that when simply mixed with existing water models, reproduced adsorption energies from vdW-DF calculations.

Interaction energies from DFT calculations were also used to re-parameterise force fields for water-gold interactions. We optimised $\mathrm{Au}-\mathrm{O}$ and $\mathrm{Au}-\mathrm{H}$ interactions for the water-gold interaction, that were used without mixing rules so that the bulk water properties were unchanged. This improved the agreement between classical and DFT adsorption energies significantly. We also found that Morse and Buckingham potentials are better suited than LJ potentials for this purpose due to their softer repulsion and longer-ranged attraction for the Au-O term. However, force fields optimised in this way can not be extended to other atomic types by mixing of parameters, due to the lack of suitable mixing rules for Morse potentials.

In MD simulations gold atoms are typically modelled as single neutral beads interacting via LJ $12-6$ potentials with no further constraints. It is well-known that this single-particle LJ representation is not capable of reproducing the polarizability of gold and hence no image charge effects are observable. ${ }^{50}$ Polarisation can be included using more complex models, such as GolP, where a gold atom is represented by two opposite charges of equal magnitude connected by a stiff rod. ${ }^{15}$ However, this doubles the number of interaction centers for gold making the simulation substantially more expensive. Another approach to simulate the 
image charge effect is a capacitance-polarizability force field which was recently developed and applied to a gold nano particle. ${ }^{51}$ In this approach partial charges of gold atoms are calculated and updated regularly during the simulation. The lack of implementation in commonly used MD code and additional computational cost might limit the application of this model. Other methods have also been developed to include image charges for metal surface interactions, such as inclusion of explicit image charges, ${ }^{45}$ representation of charges by overlapping Gaussian functions ${ }^{46,52}$ and an extended Ewald method. ${ }^{53}$ Although it is still unclear how significant polarisation effects are in the interaction between biomolecules and gold surfaces, ${ }^{54,55}$ we do note that our optimisation of PEFs was based on vdW-DFT calculations so polarisation effects are implicitly included in the effective interaction.

The second well known problem of force field approaches is that the preferential adsorption site is on the hollow and bridge sites instead of the top site as predicted by DFT and experiment. ${ }^{38}$ This issue was also tackled by Iori et al. in the GolP force field by placing virtual sites in hollow positions inside the gold surface ${ }^{15}$ and non-bonded interactions were applied between virtual sites and other atoms only. The drawback of this approach is again the increased number of particles and requirement of additional constraints. To obtain the correct adsorption site of water on the gold surface the model could be extended towards inclusion of three-body terms, as implemented for a study on water ${ }^{56}$ and studies of water on metal surfaces. ${ }^{46,57}$ In this way the adsorption site problem may be solved with a moderate computational cost and without limitation to distinct gold surface geometries. However, we note that this type of functional form is not implemented in some molecular dynamics codes, such as GROMACS, and would require the use of alternative simulation software.

We also investigated whether a force field optimised for a $\mathrm{Au}(111)$ surface would be suitable for a $\mathrm{Au}(001)$ surface, or vice versa. We found that force fields optimised using the two different surfaces were very similar. This is particularly true for Morse potentials. This has the important outcome that a single gold-water force field may be applied to both the $\mathrm{Au}(111)$ and $\mathrm{Au}(001)$ surfaces, which are two facets of gold nanoparticles. ${ }^{58}$ Thus 
a transferable force field is important for the rational design of systems containing gold nanoparticles in solution, although edges and vertices would also need to be taken into account.

Finally we ran MD simulations of water confined between two gold surfaces for the nine different force fields. These simulations showed that the force field choice has a major effect on the quantitative outcome. By comparing the structural and dynamic properties of water on the $\mathrm{Au}(111)$ and $\mathrm{Au}(001)$ surfaces we observed that the water structure is more ordered and dynamics is slower on $\mathrm{Au}(001)$ than on $\mathrm{Au}(111)$. This distinction is reduced in simulations with softer Buckingham and Morse potentials compared to LJ simulations. Simulations with fitted Buckingham and Morse potentials were able to yield structural ensembles which are in better agreement with AIMD results than any of the LJ force fields.

Since computational cost is an important consideration, and image charges and site dependence of water may be less important when modeling large biomolecules, it is likely that pair potentials will continue to be used in the forseeable future. Therefore, it is important to optimise interfacial pair potentials to give the best performance as the relative interactions strengths of different molecules are likely to play a significant role. We found that the use of alternative softer and longer-ranged pair potentials provide a promising basis for a simple and transferable force field for gold-soft matter interfaces. In the future, this approach for force

field development could be extended to biomolecular systems to investigate the influence of different gold species (surfaces/nano particles) on their thermodynamic properties. This will contribute significantly to research fields, such as biochemistry and material science, and will help to elucidate mechanistic details on the atomistic scale.

\section{Acknowledgement}

A.B. thanks the European Union for support granted in scope of the Erasmus + programme. The authors acknowledge the Collaborative Research Center 969 (Deutsche Forschungsge- 
meinschaft) and the University of Konstanz for support given through the Scientific Compute Cluster.

\section{Supporting Information Available}

The following files are available free of charge.

- si.pdf: Supplementary Information with additional Figures and Tables.

This material is available free of charge via the Internet at http://pubs.acs.org/.

\section{References}

(1) Russell, B. A.; Jachimska, B.; Komorek, P.; Mulheran, P.; Chen, Y. Lysozye encapsulated gold nanoclusters: effects of cluster synthesis on natural protein characteristics. Phys. Chem. Chem. Phys. 2017, 19, 7228-7235.

(2) Wu, C.-D.; Kuo, L.-M.; Lin, S.-J.; Fang, T.-H.; Hsieh, S.-F. Effects of temperature, size of water droplets, and surface roughness on nanowetting properties investigated using molecular dynamics simulation. Comput. Mater. Sci. 2012, 53, 25-30.

(3) Yuan, Q.; Zhao, Y.-P. Precursor Film in Dynamic Wetting, Electrowetting, and ElectroElasto-Capillarity. Phys. Rev. Lett. 2010, 104, 246101.

(4) Peter, C.; Kremer, K. Multiscale simulation of soft matter systems - from the atomistic to the coarse-grained level and back. Soft Matter 2009, 5, 4357-4366.

(5) Jorgensen, W. L.; Tirado-Rives, J. The OPLS optimized potentials for liquid simulations potential functions for proteins, energy minimizations for crystals of cyclic peptides and crambin. J. Am. Chem. Soc. 1988, 110, 1657-1666. 
(6) Jorgensen, W. L.; Maxwell, D. S.; Tirado-Rives, J. Development and Testing of the OPLS All-Atom Force Field on Conformational Energetics and Properties of Organic Liquids. J. Am. Chem. Soc. 1996, 118, 11225-11236.

(7) Sun, H. COMPASS: An ab Initio Force-Field Optimized for Condensed-Phase ApplicationsOverview with Details on Alkane and Benzene Compounds. J. Phys. Chem. B 1998, 102, 7338-7364.

(8) Piana, S.; Lindorff-Larsen, K.; Shaw, E., D. How Robust Are Protein Folding Simulations with Respect to Force Field Parameterization? Biophys. J. 2011, 100, L47-L49.

(9) Andrews, C. T.; Elcock, A. H. Molecular Dynamics Simulations of Highly Crowded Amino Acid Solutions: Comparisons of Eight Different Force Field Combinations with Experiment and with Each Other. J. Chem. Theory Comput. 2013, 9, 4585-4602.

(10) Heinz, H.; Lin, T.-J.; Kishore Mishra, R.; Emami, F. S. Thermodynamically Consistent Force Fields for the Assembly of Inorganic, Organic, and Biological Nanostructures: The INTERFACE Force Field. Langmuir 2013, 29, 1754-1765.

(11) Cicero, G.; Calzolari, A.; Corni, S.; Catellani, A. Anomalous Wetting Layer at the $\mathrm{Au}(111)$ Surface. J. Phys. Chem. Lett. 2011, 2, 2582-2586.

(12) Velasco-Velez, J.-J.; Wu, C. H.; Pascal, T. A.; Wan, L. F.; Guo, J.; Prendergast, D.; Salmeron, M. The structure of interfacial water on gold electrodes studied by x-ray absorption spectroscopy. Science 2014, 346, 831-834.

(13) Pedroza, L. S.; Poissier, A.; Fernández-Serra, M.-V. Local order of liquid water at metallic electrode surfaces. J. Chem. Phys. 2015, 142, 034706.

(14) Sushko, M. L.; Gal, A. Y.; Shluger, A. L. Interaction of Organic Molecules with the TiO2 (110) Surface: Ab Inito Calculations and Classical Force Fields. J. Phys. Chem. B 2006, 110, 4853-4862. 
(15) Iori, F.; Di Felice, R.; Molinari, E.; Corni, S. GolP: An atomistic force-field to describe the interaction of proteins with $\mathrm{Au}(111)$ surfaces in water. J. Comput. Chem. 2009, 30, 1465-1476.

(16) Wright, L. B.; Rodger, P. M.; Corni, S.; Walsh, T. R. GolP-CHARMM: First-Principles Based Force Fields for the Interaction of Proteins with $\mathrm{Au}(111)$ and $\mathrm{Au}(100)$. J. Chem. Theory Comput. 2013, 9, 1616-1630.

(17) Herbers, C. R.; Johnston, K.; van der Vegt, N. F. A. Modelling molecule-surface interactions-an automated quantum-classical approach using a genetic algorithm. Phys. Chem. Chem. Phys. 2011, 13, 10577-10583.

(18) Johnston, K.; Harmandaris, V. Properties of Benzene Confined between Two Au(111) Surfaces Using a Combined Density Functional Theory and Classical Molecular Dynamics Approach. J. Phys. Chem. C 2011, 115, 14707-14717.

(19) Johnston, K.; Herbers, C. R.; van der Vegt, N. F. A. Development of Classical MoleculeSurface Interaction Potentials Based on Density Functional Theory Calculations: Investigation of Force Field Representability. J. Phys. Chem. C 2012, 116, 19781-19788.

(20) Rosa, M.; Corni, S.; Di Felice, R. Enthalpy-Entropy Tuning in the Adsorption of Nucleobases at the $\mathrm{Au}(111)$ Surface. J. Chem. Theory Comput. 2014, 10, 1707-1716.

(21) Nadler, R.; Sanz, J. F. Effect of dispersion correction on the Au(111)-H2O interface: A first-principles study. J. Chem. Phys. 2012, 13\%, 114709.

(22) Giannozzi, P.; Baroni, S.; Bonini, N.; Calandra, M.; Car, R.; Cavazzoni, C.; Davide Ceresoli,; Chiarotti, G. L.; Cococcioni, M.; Dabo, I.; Corso, A. D.; Gironcoli, S. d.; Fabris, S.; Fratesi, G.; Gebauer, R.; Gerstmann, U.; Gougoussis, C.; Anton Kokalj,; Lazzeri, M.; Martin-Samos, L.; Marzari, N.; Mauri, F.; Mazzarello, R.; Stefano Paolini,; Pasquarello, A.; Paulatto, L.; Sbraccia, C.; Scandolo, S.; Sclauzero, G.; Seitsonen, A. P.; 
Smogunov, A.; Umari, P.; Wentzcovitch, R. M. QUANTUM ESPRESSO: a modular and open-source software project for quantum simulations of materials. J. Phys.: Condens. Matter 2009, 21, 395502.

(23) Dion, M.; Rydberg, H.; Schroeder, E.; Langreth, D. C.; Lundqvist, B. I. Van der Waals Density Functional for General Geometries. Phys. Rev. Lett. 2004, 92, 246401.

(24) Thonhauser, T.; Cooper, V. R.; Li, S.; Puzder, A.; Hyldgaard, P.; Langreth, D. C. Van der Waals density functional: Self-consistent potential and the nature of the van der Waals bond. Phys. Rev. B 2007, 76, 125112.

(25) Roman-Perez, G.; Soler, J. M. Efficient Implementation of a van der Waals Density Functional: Application to Double-Wall Carbon Nanotubes. Phys. Rev. Lett. 2009, 103, 096102.

(26) Marzari, N.; Vanderbilt, D.; De Vita, A.; Payne, M. C. Thermal Contraction and Disordering of the $\mathrm{Al}(110)$ Surface. Phys. Rev. Lett. 82, 3296-3299.

(27) Perdew, J. P.; Burke, K.; Ernzerhof, M. Generalized Gradient Approximation Made Simple. Phys. Rev. Lett. 1996, 77, 3865-3868.

(28) Gale, J. D.; Rohl, A. L. The General Utility Lattice Program (GULP). Mol. Simul. 2003, 29, 291-341.

(29) Fletcher, R.; Powell, M. J. D. A Rapidly Convergent Descent Method for Minimization. Comput. J. 1963, 6, 163-168.

(30) Shanno, D. F. Conditioning of quasi-Newton methods for function minimization. Math. Comput. 1970, 24, 647-656.

(31) Pronk, S.; Páll, S.; Schulz, R.; Larsson, P.; Bjelkmar, P.; Apostolov, R.; Shirts, M. R.; Smith, J. C.; Kasson, P. M.; Spoel, D. v. d.; Hess, B.; Lindahl, E. GROMACS 4.5: a 
high-throughput and highly parallel open source molecular simulation toolkit. Bioinformatics 2013, btt055.

(32) Essmann, U.; Perera, L.; Berkowitz, M. L.; Darden, T.; Lee, H.; Pedersen, L. G. A smooth particle mesh Ewald method. J. Chem. Phys. 1995, 103, 8577-8593.

(33) Bussi, G.; Donadio, D.; Parrinello, M. Canonical sampling through velocity rescaling. J. Chem. Phys. 2007, 126, 014101.

(34) Heinz, H.; Vaia, R. A.; Farmer, B. L.; Naik, R. R. Accurate Simulation of Surfaces and Interfaces of Face-Centered Cubic Metals Using 12-6 and 9-6 Lennard-Jones Potentials. J. Phys. Chem. C 2008, 112, 17281-17290.

(35) Berendsen, H.; Grigera, J.; Straatsma, T. The missing term in effective pair potentials. J. Phys. Chem. 1987, 91, 6269-6271.

(36) Neria, E.; Fischer, S.; Karplus, M. Simulation of activation free energies in molecular systems. J. Chem. Phys. 1996, 105, 1902-1921.

(37) Parrinello, M.; Rahman, A. Polymorphic transitions in single crystals: A new molecular dynamics method. J. Appl. Phys. 1981, 52, 7182-7190.

(38) Verdaguer, A.; Sacha, G. M.; Bluhm, H.; Salmeron, M. Molecular Structure of Water at Interfaces: Wetting at the Nanometer Scale. Chem. Rev. 2006, 106, 1478-1510.

(39) Velasco-Velez, J.-J.; Wu, C. H.; Pascal, T. A.; Wan, L. F.; Guo, J.; Prendergast, D.; Salmeron, M. The structure of interfacial water on gold electrodes studied by x-ray absorption spectroscopy. Science 2014, 346, 831-834.

(40) Michaelides, A.; Ranea, V. A.; de Andres, P. L.; King, D. A. General Model for Water Monomer Adsorption on Close-Packed Transition and Noble Metal Surfaces. Phys. Rev. Lett. 2003, 90, 216102. 
(41) Liu, R. Adsorption and dissociation of H2O on Au(111) surface: A DFT study. Comput. Theor. Chem. 2013, 1019, 141-145.

(42) Rosa, M.; Corni, S.; Di Felice, R. van der Waals effects at molecule-metal interfaces. Phys. Rev. B 2014, 90, 125448.

(43) Peköz, R.; Johnston, K.; Donadio, D. Tuning the adsorption of aromatic molecules on Platinum via halogenation. J. Phys. Chem. C 2014, 118, 6235-6241.

(44) Verde, A. V.; Acres, J. M.; Maranas, J. K. Investigating the Specificity of Peptide Adsorption on Gold Using Molecular Dynamics Simulations. Biomacromolecules 2009, $10,2118-2128$.

(45) Spohr, E. Molecular simulation of the electrochemical double layer. Electrochim. Acta 1999, 44, 1697-1705.

(46) Siepmann, J. I.; Sprik, M. Influence of surface topology and electrostatic potential on water/electrode systems. J. Chem. Phys. 1995, 102, 511-524.

(47) Mark, P.; Nilsson, L. Structure and Dynamics of the TIP3P, SPC, and SPC/E Water Models at 298 K. J. Phys. Chem. A 2001, 105, 9954-9960.

(48) Krynicki, K.; Green, C. D.; Sawyer, D. W. Pressure and temperature dependence of self-diffusion in water. Faraday Discuss. Chem. Soc. 1978, 66, 199.

(49) Kumar, P.; Franzese, G.; Buldyrev, S. V.; Stanley, H. E. Molecular dynamics study of orientational cooperativity in water. Phys. Rev. E 2006, 73, 041505.

(50) Iori, F.; Corni, S. Including image charge effects in the molecular dynamics simulations of molecules on metal surfaces. J. Comput. Chem. 2008, 29, 1656-1666.

(51) Li, X.; Ågren, H. Molecular Dynamics Simulations Using a Capacitance-Polarizability Force Field. J. Phys. Chem. C 2015, 119, 19430-19437. 
(52) Guymon, C. G.; Rowley, R. L.; Harb, J. N.; Wheeler, D. R. Simulating an electrochemical interface using charge dynamics. Condens. Matter Phys. 2005, 8, 335-356.

(53) Takae, K.; Onuki, A. Applying electric field to charged and polar particles between metallic plates: Extension of the Ewald method. J. Chem. Phys. 2013, 139, 124108.

(54) Piana, S.; Bilic, A. The Nature of the Adsorption of Nucleobases on the Gold (111) Surface. J. Phys. Chem. B 2006, 110, 23467-23471.

(55) Bilić, A.; Reimers, J. R.; Hush, N. S.; Hafner, J. Adsorption of ammonia on the gold (111) surface. J. Chem. Phys. 2002, 116, 8981-8987.

(56) Molinero, V.; Moore, E. B. Water Modeled As an Intermediate Element between Carbon and Silicon. J. Phys. Chem. B 2009, 113, 4008-4016.

(57) Willard, A. P.; Reed, S. K.; Madden, P. A.; Chandler, D. Water at an electrochemical interface-a simulation study. Faraday Discuss. 2009, 141, 423-441.

(58) Hughes, Z. E.; Walsh, T. R. Non-covalent adsorption of amino acid analogues on noblemetal nanoparticles: influence of edges and vertices. Phys. Chem. Chem. Phys. 2016, $18,17525-17533$. 
Graphical TOC Entry

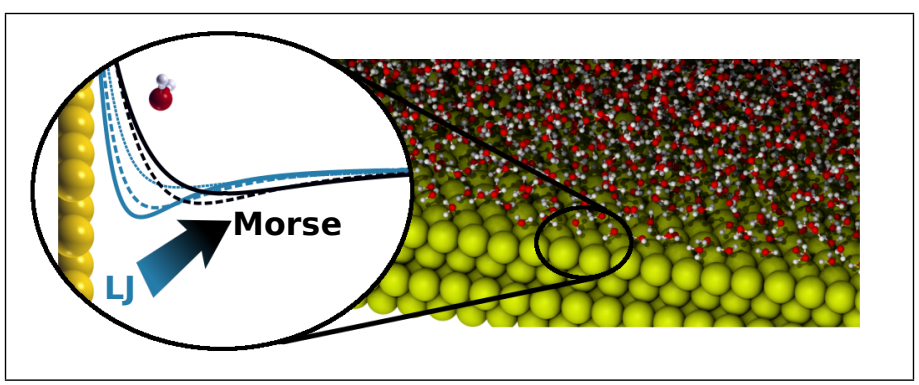

